\title{
Les sciences humaines dans les parcours scientifiques et techniques professionnalisants : quelles finalités et quelles modalités pratiques? Présentation générale
}

\author{
Comité éditorial : Alain Bernard ${ }^{1}$, Michèle Dell'Angelo², Sandrine de Montgolfier ${ }^{3}$, \\ Anne-Sophie Godfroy ${ }^{4}$, Michaël Huchette ${ }^{2}$, Arnaud Mayrargue ${ }^{5}$ et Camille Roux ${ }^{6}$
}

${ }^{1}$ UPEC-ESPE de Créteil, Centre A. Koyré (MNHN-CNRS-EHESS, UMR8560), labex HASTEC (ANR-10-LABX-85), France

${ }^{2}$ UPEC-ESPE de Créteil, STEF (ENS Cachan-Ifé ENS Lyon), France

${ }^{3}$ UPEC-ESPE de Créteil, IRIS (CNRS-Inserm-EHESS, UMR8156), France

${ }^{4}$ UPEC-ESPE de Créteil, SND (Université Paris-Sorbonne-CNRS, FRE 3593), France

${ }^{5}$ UPEC-ESPE de Créteil, SPHERE (Université Paris Diderot-CNRS, UMR 7219), France

${ }^{6}$ UPEC-UFR de sciences, STEF (ENS Cachan-Ifé ENS Lyon), France

\section{Présentation générale :}

0 . Origine et contexte de ce volume

0.1 Le thème du recueil et ses grandes problématiques

0.2 L'origine et l'originalité du recueil

0.3 . Le poids du contexte institutionnel et de ses évolutions récentes pour l'actualité de la thématique

0.4 La préparation et l'organisation du recueil

1. Approches comparatistes

1.1 Présentation générale : quatre contributions illustrant un contexte institutionnel favorable aux comparaisons

1.2 Trois approches pour la formation en sciences humaines et sociales dans les écoles d'ingénieurs

1.3. Du comparatisme à la définition d'approches prospectives

2. Influence du contexte local

2.1 Présentation générale : quatre études de cas illustrant des collaborations locales

2.2 Les expériences comparées du Polytech Nantes, de l'UTBM et de l'Ecole Centrale de Lyon

2.3 L'exploration des potentialités d'une expérience collaborative dans l'université Catholique de Lille et à l'université Paris Est Créteil

2.4 Conclusions prospectives : la redéfinition des sciences humaines et sociales en contexte professionnalisant, et la fédération locale de communautés d'enseignants-chercheurs autour de projets communs

3. Dispositifs pédagogiques unrestricted use, distribution, and reproduction in any medium, provided the original work is properly cited. 


\section{SHS Web of Conferences}

4. Formation et recherche

4.1 Le développement concomitant d'une activité de recherche et de formation : un paradigme utile pour analyser les trois contributions de cette partie

4.2 Trois parcours «d'enseignement-recherche »

4.3 Trois similitudes de ces études : leur caractère génétique, le poids des circonstances institutionnelles, le caractère interdisciplinaire des champs de recherche

4.4 La question originale de l'ambivalence des objets de recherche, vus comme « objets pédagogiques transitionnels »

5. Fondements, enjeux et perspectives

5.1 La raison d'être de réflexions pluralistes sur des enjeux fondamentaux des objets étudiés dans ce volume

5.2 Quatre systèmes critiques débouchant sur des hypothèses fortes et de nouveaux points de vue sur la conception de formations professionnelles

5.3 Points de convergence et perspectives

Résumé. Cette introduction a été rédigée par les membres du comité éditorial, également membres du groupe «Sciences et Techniques en interférences ${ }^{1}$ de l'Université ParisEst-Créteil (UPEC). Elle décrit d'abord l'origine de ce volume collectif, le contexte institutionnel et de recherche des questions qui y sont soulevées. Elle présente le mode de composition du recueil, notamment la manière dont la répartition thématique des différentes contributions a été obtenue. Ensuite, cinq parties correspondant aux cinq sections du volume introduisent les contributions qui y ont été rassemblées en soulignant, lorsqu'il y a lieu, les thématiques communes et les champs concernés. La première partie, Etudes comparatistes, renvoie à des études globales au niveau français ou européen. Elle est complétée par la seconde, Influence du contexte local, qui présente des études de cas plus localisées et focalisées sur l'importance du contexte institutionnel. La partie suivante, Dispositif pédagogiques, présente d'autres études de cas qui problématisent principalement l'ingénierie pédagogique mise en œuvre. La quatrième partie, Formation et Recherche, s'intéresse à trois modes originaux d'articulation entre formation professionnelle et initiation à la recherche. Enfin la dernière partie, Fondements et Enjeux, rassemble des contributions à caractère philosophique, sociologique et historique interrogeant les enjeux fondamentaux sous-jacents à la conception des formations qui font l'objet du volume.

\section{Origine et contexte de ce volume ${ }^{2}$}

\subsection{Le thème du recueil et ses grandes problématiques}

En France comme à l'étranger, la formation universitaire de futurs professionnels, notamment celle des enseignants, médecins, ingénieurs et techniciens, comprend très souvent des unités d'enseignement qui visent à introduire une mise en perspective par les sciences humaines et sociales : philosophie, épistémologie, éthique, histoire des sciences et des techniques, didactique des sciences et techniques, sociologie ou anthropologie, lettres et langues.

Dans le cas français, qui intéresse la plupart des contributions de ce volume, des modules ont été intégrés dans ces cursus professionnalisants selon des temporalités et des histoires différentes, ce qui

\footnotetext{
${ }^{1}$ Voir carnet de recherche : http: //interferences .hypotheses . org

2 Par simplicité nous référons aux articles de ce volume par la référence chiffrée employée par l'éditeur pour identifier les contributions : elle renvoie à la section dans laquelle ils se trouvent, et leur numéro d'ordre dans la section, sous la forme par exemple « $05002 »$, pour le 2nd article de la 5ème section.
} 
leur donne des contours extrêmement divers. En outre, s'il existe une réflexion, elle s'est généralement engagée de manière éclatée suivant les lieux et les institutions, y compris pour un même domaine de formation. Font toutefois exception les formations aux professions médicales et de la santé, qui ont ressenti depuis déjà vingt ans la nécessité d'engager une réflexion collective (Visier, 2011) : cette dernière a conduit à une mutualisation des conceptions de modules d'enseignement et des ressources associées. La plupart du temps, il n'existe cependant pas de pratique normalisée ni de consensus évident. Par contre, sous l'égide de différentes institutions et sociétés savantes, des échanges ont permis une confrontation des approches pédagogiques. Elles ont permis dans certains cas de faire émerger de premières thématiques de recherches portant sur ces innovations : ce volume, issu lui-même d'un colloque parrainé entre autre par la Société Française d'Histoire des Sciences et des Techniques ${ }^{3}$, entend contribuer au mouvement de réflexion et de théorisation de ce domaine éclectique, en adoptant le plus souvent, comme on le verra, une position comparatiste.

En termes de recherche, on peut distinguer plusieurs niveaux de questionnement possibles, tous illustrés par les contributions ici réunies. Au niveau des enjeux scientifiques, politiques et sociétaux liés au développement de ce type d'enseignement, on constate que la prise de distance critique (historique, épistémologique, didactique, sociologique ou anthropologique) attendue dans les cursus universitaires de formation professionnelle en sciences et techniques, est devenue, depuis plus ou moins longtemps, une injonction institutionnelle jusqu'au niveau européen, voire mondial. Au-delà de cette injonction, si ces discours énoncent volontiers que ces enseignements sont souhaitables et qu'ils constituent une mission «naturelle » des institutions qui participent à la formation professionnelle, une prise de distance critique reste nécessaire pour ne pas en rester là et questionner ce type de présupposés : elle conduit alors s'interroger sur les enjeux politiques, sociétaux, éthiques, sous-jacents à ces injonctions, ainsi que sur les évolutions historiques souvent complexes qui ont conduit à de tels impératifs ${ }^{4}$.

Au niveau des modalités d'enseignement, il existe depuis maintenant longtemps dans le monde anglo-saxon, et de manière plus récente en France, des recherches nombreuses sur la pédagogique universitaire (Colet, McAlpine, Fanghanel, \& Weston, 2011). Dans ce vaste champ, la conception et la mise en œuvre d'enseignements en sciences humaines pour la formation de futurs professionnels en sciences et technologie possèdent des spécificités qui méritent d'être étudiées. Sans prétention d'exhaustivité, elles touchent aux questions suivantes, difficilement dissociables en pratique mais qu'il est utile de distinguer pour les besoins de l'analyse : (a) la conception de ressources documentaires, notamment (mais pas exclusivement) secondées par des outils numériques ${ }^{5}$; (b) les modalités de lecture et d'exploration de ces ressources dans un contexte de formation professionnelle ${ }^{6}$; (c) les stratégies globales de conception et d'ingénierie de formation associées à de tels enseignements ${ }^{7}$, (d) l'articulation plus ou moins prononcée entre les enseignements de sciences humaines et sociales et les enseignements scientifiques et techniques stricto sensu, pour autant que cette clause ait un sens dans le contexte étudié ; ${ }^{8}$ enfin (e) l'articulation entre le contenu même de ce type d'enseignement avec des lieux extrauniversitaires : on pense au premier chef aux lieux d'exercice professionnels bien évidemment (stages

\footnotetext{
3 Voir plus bas $\$ 0.2$.

${ }^{4}$ Dans ce volume, les contributions réunies dans la section 5 «Fondements et enjeux » renvoient toutes à ce type de problématique, sous des approches toutefois différentes.

${ }^{5}$ Dans ce volume, voir les trois contributions réunies dans la partie 4 «Formation et recherche », particulièrement les deux premières pour ce qui concerne les projets d'humanités numériques (Bonah et Danet 04001, Laubé et Chambon 04002). Voir aussi, dans la partie 5, la contribution de Husson et alii 05004, et dans la partie 2 «Influence du contexte local » la contribution de Bernard et alii. 02004.

6 Voir ici les contributions de Bonah et Danet à nouveau (04001), Gosztonyi (04003), Husson et alii (05004).

${ }^{7}$ Les études de cas réunies dans la section 3 « Dispositifs pédagogiques » explorent cette question, elle est également abordée au niveau des principes mêmes de conception de formation dans les contributions de la partie 5 «fondements et enjeux », ainsi que de manière comparatiste dans la section 1 « approches comparatistes ».

${ }^{8}$ La plupart des contributions de ce volume abordent ce point, notamment toutes celles qui concernent la formation en écoles d'ingénieurs pour tout ce qui touche au projet de fin d'étude.
} 
en entreprise, en hôpital, ou en établissement scolaire), mais aussi aux lieux muséaux ou dédiés à la culture scientifique, aux laboratoires ou aux instituts scientifiques ${ }^{9}$.

Par ailleurs et comme toujours en matière d'enseignement, notamment d'enseignements expérimentaux ou parfois marginaux, une voie de compréhension et d'analyse passe moins par un questionnement théorique et de recherche, que par des récits problématisés d'expériences pédagogiques. Plusieurs des contributions de ce volume relèvent a priori davantage de ce genre que de celui d'un exposé de recherche ${ }^{10}$. Ce genre narratif et circonstancié est inévitable dans le contexte encore très mouvant et non stabilisé qui, en France du moins, caractérise plusieurs des milieux d'enseignants visés, comme nous l'avons rappelé plus haut, en l'absence d'instances de mutualisation et d'échanges, qui répondraient pourtant à une réelle demande.

Il faut en outre signaler immédiatement une difficulté plus fondamentale touchant à la distinction entre récit problématisé et exposé de recherche. En effet, une séparation stricte entre le compte-rendu d'une expérience pédagogique problématisée en profondeur et un exposé de recherche s'appuyant sur un terrain très spécifique, n'est pas toujours facile à établir. Dans certains cas, notamment celui où projet de recherche et projet de conception d'enseignements à vocation professionnalisante vont de pair, on verra plus loin $^{11}$ que la perspective génétique offre une entrée naturelle pour appréhender cette articulation.

\subsection{L'origine et l'originalité du recueil}

Le recueil est issu pour l'essentiel d'une rencontre de deux jours organisée en février 2013 à l'université Paris-Est Créteil (UPEC) par plusieurs enseignants chercheurs de l'IUFM $^{12}$ de Créteil, aujourd'hui ESPE de l'Académie de Créteil, et de la faculté de Sciences et technologie ${ }^{13}$, et réunis sous la bannière d'un axe de recherche collaboratif intitulé « sciences et techniques en interférences ${ }^{14}$. L'appel d'offre de cette conférence ${ }^{15}$ énonçait, sous forme de questions, les grandes thématiques qui ont été présentées ci-dessus (\$0.1). L'argumentaire de cette rencontre, comme celui de ce volume qui le prolonge et l'amplifie, présentait l'originalité évidente de proposer un croisement de perspectives entre des champs d'enseignements semblables par le rattachement générique (les sciences humaines et sociales) mais dans des secteurs de professionnalisation qui communiquent peu entre eux : les milieux de formation d'ingénieurs et de techniciens, les milieux de formation d'enseignants, enfin les milieux de formation de médecins et de personnels de santé ${ }^{16}$. De l'aveu de nombreux participants à cette manifestation, cette expérience a été un succès indubitable en raison précisément de cet effort de rencontre, qui représentait pour eux comme pour nous, une ouverture inédite et un élargissement de perspective. Si en effet, et sans surprise, les divergences dues aux différences de contexte apparaissaient clairement, bien des participants ont été frappés de la similitude des questionnements et plusieurs en ont tiré immédiatement des leçons.

\footnotetext{
${ }^{9}$ Ici, la contribution de Laubé et Chambon (04002), la seule à évoquer la professionnalisation vers les métiers de médiation, insiste tout particulièrement sur ce problème, à travers une étude de cas.

${ }^{10}$ C'est le cas notamment des études de cas réunies dans les sections 2 « Influences du contexte local », 3 « Dispositif pédagogiques » et 4 « Formation et recherche».

11 Dans l'introduction de la section 4 « formation et recherche », §4.

12 Instituts Universitaires de Formation des Maîtres, qui ont été remplacés depuis par les Ecoles Supérieurs du Professorat et de l'Education (ESPE).

13 Elles sont toutes deux composantes de l'UPEC.

${ }^{14}$ Les circonstances qui ont initié ce groupe sont relatées dans ce volume dans la contribution d'Alain Bernard, Sandrine de Montgolfier et Camille Roux (02004); elles sont liées à ce qui était alors la préparation du futur projet d'ESPE. Il possède par ailleurs un carnet de recherche consultable à l'adresse http://interferences .hypotheses.org/ (consulté le 25.9.14).

15 Le thème, l'appel d'offre, ainsi que le programme détaillé de la conférence sont toujours consultables sur son site http://shst2013-upec.sciencesconf .org/ (consulté le 25.9.14).

${ }^{16}$ L'une des contributions, celle de S. Laubé et G. Chambon (04002), touche également à la formation des médiateurs culturels dans le domaine scientifique et technique.
} 
Cette convergence est reflétée par la forte unité thématique de ce recueil, qu'il a été possible de structurer plus fortement que s'il s'était agi simplement des actes du colloque en question. La moitié environ des contributeurs du colloque a proposé un article qui a été retenu par le comité scientifique ${ }^{17}$. Le processus éditorial nous a confirmé qu'il était possible d'approfondir les comparaisons, comme en témoigneront les introductions thématiques proposées ci-dessous.

Nous avons déjà fait allusion plus haut à une autre surprise intéressante de ce colloque, à savoir, qu'il n'a pas été possible de différencier aussi nettement que nous le pensions au départ, les récits d'expériences pédagogiques, des articles de recherche. Avec le recul, nous en avons conclu que ce mélange avait plusieurs raisons probables. Une première tient à l'évolution temporelle : les expériences pédagogiques existent pour la plupart depuis un temps suffisamment long pour qu'il soit maintenant possible de substituer à la simple narration une attitude réflexive permettant de nourrir les recherches. Le mélange s'explique encore par une raison qui fait aussi l'une des originalités du recueil : à quelques exceptions près, la plupart des contributeurs n'envisageait pas leur contribution dans une perspective de recherche au sens où l'on parle aujourd'hui des recherches sur la pédagogie dans l'enseignement supérieur. Dans un esprit qui est au fond proche du Scholarship of Teaching and Learning (SOTL) du monde anglo-saxon, beaucoup ont essayé d'appréhender les problèmes les plus profonds que posaient leurs enseignements, au niveau de ses enjeux comme de ses modalités, à partir de l'outillage conceptuel et scientifique qui leur était le plus familier. La diversité et la richesse des approches qui en résulte, est une originalité de ces études et dans bien des cas ouvre des perspectives nouvelles et intéressantes, comme on le verra plus bas. Enfin il existe des raisons plus structurelles sur lesquelles nous reviendrons plus bas, en introduction de la section « Formation et recherche».

La dernière caractéristique du colloque comme du recueil n'est pas vraiment une originalité ni une surprise, mais elle est importante à souligner car elle induit un biais aussi intéressant que révélateur. La majorité des contributeurs au colloque, comme des auteurs retenus pour ce recueil, travaillent en effet dans les milieux de formation d'ingénieurs. Il y a bien sûr à cela des explications circonstancielles. Ainsi, les enseignants français de sciences humaines et sociales qui interviennent dans les milieux de la santé bénéficient déjà d'un rattachement institutionnel fort qui leur permet rencontres, échanges ou publications ${ }^{18}$, du moins pour nombre d'entre eux ${ }^{19}$. Les enseignants intervenant dans la formation des enseignants de sciences et techniques étaient quant à eux, à l'époque du colloque, submergés pour beaucoup d'entre eux par la préparation de la réforme qui a conduit à la création des ESPE. Mais au-delà de ces explications, il faut aussi prendre en compte les raisons historiques et structurelles qui ont conduit les milieux de formation d'ingénieurs à réfléchir à ces questions et le manque de lieux institutionnels qui leur sont disponibles pour de telles discussions.

\subsection{Le poids du contexte institutionnel et de ses évolutions récentes pour l'actualité de la thématique}

Puisque la plupart des contributions de ce recueil concernent le cas français (§0.1), il faut ici rappeler l'importance et l'actualité institutionnelle de ces questions en France. Ces évolutions récentes ne sont à vrai dire pas dissociables du contexte international, particulièrement européen, dans le cadre de l'harmonisation des structures universitaires et, plus récemment, de la montée du thème « Responsible

\footnotetext{
17 Voir plus loin le mode de curation et de composition de l'ouvrage ( $\$ 0.4)$.

18 Il s'agit du collège des enseignants de sciences humaines et sociales en médecine et santé, qui a tenu sa dernière rencontre annuelle « Discours, récits en santé » à Amiens en juin 2013, quelques mois après notre colloque.

http://collegeshsenmedecine.etud.univ-montp1.fr/congres-et-colloques/congres-2013/

(consulté le 25.9.14). Nous remercions à nouveau Christian Bonah d'avoir bien voulu contribuer à cette rencontre à l'UPEC par une conférence plénière récapitulant l'historique et les travaux de ce collège, dont il a été le premier président. http://shst2013-upec.sciencesconf .org/12639 (consulté le 25.9.14).

19 Tous en effet ne sont pas membres du collège.
} 
Research and Innovation ${ }^{20}$. Elles bousculent aujourd'hui l'ensemble des institutions d'enseignement supérieur, comme nous allons le rappeler.

Concernant la formation des enseignants, la récente réforme du système de formation, dont les principes ont été débattus en 2012 pour une mise en application en $2013^{21}$, prolonge et amplifie les principes qu'avait déjà posés la précédente réforme du système de formation, en 2008-9. Cette évolution implique la mise en place de masters spécifiquement orientés vers la formation à l'enseignement, et a conduit à réfléchir à la formation à ou par la recherche que devraient y recevoir les étudiants. L'article 7 de l'arrêté de juillet 2013 instituant les masters MEEF précise ainsi que la formation « s'appuie sur une activité d'initiation à la recherche » puis que "l'activité de recherche doit, au-delà du contenu disciplinaire, permettre l'acquisition de compétences en lien avec le métier d'enseignant ou de personnel d'éducation (... .). ». Ce qui a été ainsi inscrit dans la loi est la conséquence d'une réflexion qui avait été entamée dans les formations mises en place lors de la précédente réforme, conduisant à repenser la place de la recherche dans les formations, notamment au travers de la rédaction du mémoire de master des étudiants ${ }^{22}$. Ce rapprochement avec le monde de la recherche mobilise très fortement les champs des sciences humaines et sociales, qui doivent se positionner désormais comme partie intégrante d'une formation par la recherche, et non plus seulement comme « outillage » spécifique à destination d'instituts de formation ayant une culture et une autonomie propres, en partie distinctes des composantes universitaires habituelles.

Concernant la formation des futurs médecins et personnels de santé, les analyses (Visier, 2011) montrent une temporalité en partie similaire à celle de la formation des enseignants, mais une vingtaine d'années auparavant. Les premières réformes ayant permis d'introduire des sciences humaines et sociales dans le cursus remontent à 1992. Les deux différences notables avec la façon dont la formation des enseignants s'est reconstruite sont d'une part que la réforme du cursus s'est faite à partir des premières années du curriculum vers les dernières, et d'autre part que le niveau master (second cycle) a été touché plus tôt puisque les premières réformes introduisant la possibilité de valider des certificats de maîtrise remontent à 98, et le passage aux masters à 2003 - soit avant les réformes évoquées ci-dessus (Visier, 2011, p. 147).

Ces évolutions relativement récentes doivent à leur tour se comprendre dans le contexte plus large des évolutions des missions et de la structuration de l'université. En France, là encore, et dans le sillage du processus de Bologne, les lois LRU ${ }^{23}$ de 2007 amplifiées et prolongées récemment par la loi $\mathrm{ESR}^{24}$, ont reconnu pour la première fois une mission d'insertion professionnelle aux universités, et le diplôme de master s'est vu reconnaître des vocations élargies. L'arrêté de 2014 sur les diplômes de licence et master, indique ainsi que «l'adossement à la recherche vaut pour toutes les formations même s'il peut prendre des formes différentes ${ }^{25}$ et précise que les parcours professionnalisants « doivent joindre savoirs et pratiques, intégrant les compétences apportées par les établissements d'enseignement

\footnotetext{
20 Voir par exemple le colloque organisé en novembre 2014 par la Présidence italienne: « Science, Innovation and Society Achieving Responsible Research and Innovation » (lien: http://www . sis-rri-conference.eu consulté le 16.10.2014) et les travaux de René von Schomberg qui a popularisé ce thème, par exemple (Von Schomberg 2013).

${ }^{21}$ Par la fondation des ESPE et la mise en place concomitante des masters de la nouvelle mention MEEF (Métiers de l'Education, de l'Enseignement de la Formation), et par un programme simultané de « refondation de l'école ».

22 Dans ce volume, plusieurs contributions sont à rapporter directement à ces évolutions récentes et au contexte de cette réflexion, ainsi la conclusion de la contribution de Muriel Guedj (01004) où un projet de recherche pensé en fonction de la construction de la professionnalité des enseignants de sciences expérimentales est brièvement présenté ; le contexte des réflexions de Sandrine de Montgolfier, Camille Roux et Alain Bernard (02004) sur un projet de recherche dont l'existence est intimement liée aux circonstances de la fondation de l'ESPE de Créteil, ou encore la contribution de Jérôme Fatet sur la formation en master MEEF des enseignants de sciences physiques et chimiques, en lien à la problématique de la réplication d'expériences historiques (03004).

23 Liberté et Responsabilité des Universités, loi d'août 2007 passée sous le ministère de Valérie Pécresse.

24 Enseignement Supérieur et Recherche, loi de juillet 2013 passée sous le ministère de Geneviève Fioraso.

25 Arrêté du janvier 2014 « fixant le cadre national des formations conduisant à la délivrance des diplômes nationaux de licence, de licence professionnelle et de master », article 17.
} 
supérieur et par les milieux économiques et sociaux. »Comme dans l'arrêté concernant les masters MEEF, le lien entre recherche et formation professionnelle est postulé mais très peu spécifié, en particulier la notion «d'adossement» ne renvoie pas spécifiquement à la formation par la recherche, mais à un éventail très large de possibilités et de pratiques d'articulation ; il n'en reste pas moins qu'il est rendu obligatoire, institutionnalisant le caractère universitaire des formations professionnelles validées par un diplôme.

Ces dispositions récentes, qui ne touchent donc que depuis deux ou trois décennies les milieux de formation des enseignants ou des métiers de la santé, doivent cependant se comprendre à l'échelle d'une histoire beaucoup plus longue, qui a concerné toutes les formations scientifiques et techniques avec des modalités diverses. La question d'articuler en général la formation des ingénieurs à des programmes de recherche remonte elle aussi à une période assez récente, mais cette évolution n'a fait en réalité qu'accélérer et consacrer un mouvement plus ancien, qui concernait déjà un certain nombre d'écoles d'ingénieurs progressivement intégrées à des universités ou ayant d'emblée un caractère universitaire, comme les universités technologiques. Les deux contributions de Michel Cotte dans ce volume (01002 et 02001), éclairent ces processus historiques et donnent deux exemples de travail en sciences humaines dans deux de ces écoles, une école Polytechnique universitaire d'une part, une université technologique de l'autre. En complément, la conclusion de son second article insiste sur la question de la nature des recrutements d'enseignants-chercheurs dans ce domaine et du lien, pour lui nécessaire, à la recherche ${ }^{26}$. Même dans les écoles qui n'affichent pas traditionnellement projet de recherche académique en sciences humaines et sociales, la question de la structuration de la recherche est vivante, comme en témoigne par exemple la conclusion de la contribution de Nicolas Hourcade (02002). La question générale, à l'échelle européenne cette fois-ci, de la constitution de projets de formation et de recherche à la fois interdisciplinaires, articulant sciences humaines et disciplines « strictement » techniques, et adaptés aux projets d'étude des étudiants, est bien vivante dans ces milieux, comme en témoigne la contribution prospective d'Anne-Sophie Godfroy (01003).

Au total, le contexte institutionnel peut donc expliquer l'actualité de la thématique et les raisons pour lesquelles des enseignants-chercheurs en IUFM puis en ESPE, écoles atteintes en dernier par le mouvement «d'universitarisation » des formations professionnalisantes, pouvaient souhaiter une telle rencontre, qui équivaut aussi à une mise en perspective dans un cadre qui, sans être strictement normatif, devient cependant de plus en plus commun, autorisant les comparaisons.

\subsection{La préparation et l'organisation du recueil}

Sur les quelques quarante contributions au colloque, une vingtaine de collègues ont envoyé une première version qui a été soumise, sous couvert d'anonymat, à un comité scientifique élargi par rapport à celui du colloque $^{27}$. Nous remercions chaleureusement, à cette occasion, les membres de ce comité scientifique élargi, qui ont fait un travail de relecture approfondi et de qualité ${ }^{28}$.

Nous avons ensuite regroupé les articles selon leurs thèmes. Ainsi, le premier groupe, intitulé Approches comparatistes (Sect. 1), présente des analyses comparatives globales dans un contexte français ou européen. Les trois parties suivantes proposent toutes des études de cas circonstanciées sur une ou au plus deux institutions particulières. La section intitulée Influences du contexte local, complète et illustre la précédente, en traitant comme son titre l'indique, de la dépendance de la nature des

\footnotetext{
${ }^{26}$ Les nombreuses contributions, dans ce volume, des collègues d'universités technologiques (Charles Lenay, Pierre Lamard, Yves-Claude Lequin 05001, Gérard Gaglio 03002, Guillaume Carnino 05002) témoignent à leur façon de la vitalité de cette question dans les universités technologiques.

27 Etant donné en effet la variété et la richesse des champs disciplinaires et des milieux de formation couverts, il était nécessaire de faire appel à de nouvelles compétences.

${ }^{28}$ La liste des membres qui ont bien voulu communiquer leur identité, conformément au vadémécum qui leur a été envoyé, est accessible dans ce volume dans la rubrique Remerciements (00001).
} 
enseignements donnés à l'environnement institutionnel. La troisième section est davantage focalisée sur la description détaillée et problématisée de Dispositifs pédagogiques. La quatrième, intitulée Formation et recherche, s'intéresse quant à elle à l'analyse de dispositifs qui conjoignent projet de recherche en sciences humaines et conception d'enseignements professionnalisants, l'accent étant mis précisément sur cette articulation entre recherche et formation. La dernière section, Fondements et enjeux, rassemble enfin les contributions qui proposent une analyse fondamentale des conditions qu'il faut étudier pour concevoir tel ou tel type d'intégration d'enseignements en sciences humaines et sociales à la formation professionnelle.

Chaque partie est introduite ci-dessous par un propos restituant le contexte de chaque contribution, les champs de recherches concernés et indique, quand cela est possible et pertinent, les points de convergence entre les contributions.

\section{Approches comparatistes}

\subsection{Présentation générale : quatre contributions illustrant un contexte institutionnel favorable aux comparaisons}

Le thème même du présent recueil invite à réfléchir sur la place et la fonction de formations en sciences humaines et sociales dans les formations professionnalisantes de futurs scientifiques, en pratiquant une comparaison raisonnée entre différentes institutions, pays ou secteurs de formation. Cette partie regroupe quatre contributions qui abordent cette question de front, alors que la section suivante regroupe des études portant pour l'essentiel sur des études de cas impliquant une, ou au plus deux, institutions. Pour ce faire, les quatre contributeurs et contributrices mettent en œuvre des approches différentes. Les trois premières s'intéressent spécifiquement aux sciences humaines dans les formations d'ingénieurs, que ce soit en France pour Catherine Roby (01001) et Michel Cotte (01002) ou en Europe pour AnneSophie Godfroy (01003) et Michel Cotte en partie. La quatrième contribution, de Muriel Guedj (01004), s'appuie sur les textes d'orientation et de réflexion sur la formation dans le domaine de l'ingénierie, de la médecine et de la santé, pour en tirer à la fois un éclairage ${ }^{29}$ et un sujet d'inspiration permettant de réfléchir sur la place, en France, des sciences humaines dans les formations d'enseignants des collèges et lycées en sciences expérimentales.

Si les formations d'ingénieur étaient de fait les mieux représentées dans le colloque qui a donné naissance au recueil, comme on l'a vu plus haut (\$0.3), cette emphase reflète aussi la situation originale de ce secteur de formation, comme le soulignent C. Roby et $\mathrm{M}$. Cotte : les formations d'ingénieur sont en général soumises en France à l'avis de la commission des titres de l'ingénieur (C.T.I.), un organisme qui joue un double rôle de lieu de débats évolutifs sur les objectifs, les enjeux et le contenu des formations d'ingénieur, et dans l'accréditation des formations en question. Comme le souligne M. Cotte, ce mélange de flexibilité et de contrainte crée les circonstances favorables pour une réflexion globale sur le métier d'ingénieur, et en particulier sur la définition dynamique du rôle et de la place des sciences humaines et sociales dans leur formation ${ }^{30}$. Le questionnement qui fait l'objet de ce recueil est donc inscrit dans les évolutions actuelles des formations d'ingénieur, dans un contexte lui-même en transformation rapide ${ }^{31}$.

On a également vu plus haut $(\S 0.3)$ que la formation en sciences humaines et sociales des futurs professionnels de la médecine et de la santé a fait elle aussi l'objet en France depuis 1992 d'une réflexion qui faisait suite à une évolution institutionnelle majeure, définissant une première obligation d'inscrire une part de sciences humaines dès la première année de formation des futurs médecins. Cette

\footnotetext{
29 Ou un « regard porté » selon les termes de l'auteur.

${ }^{30}$ Cette influence est particulièrement sensible dans les études de cas détaillées que nous avons regroupés dans la section suivante : Polytech Nantes et UTBM (M. Cotte 02001), Ecole Centrale de Lyon (N. Hourcade 02002), les écoles dépendant de l'Université Catholique de Lille (G. Aiguier, C. Didier 02003).

31 C'est ce dont témoigne la contribution prospective d'Anne-Sophie Godfroy, comme nous le verrons plus bas.
} 
institutionnalisation peut être elle aussi qualifiée de souple car elle ne définit que des orientations générales. On a vu encore qu'elle a donné naissance à un collectif d'enseignants toujours actifs, désormais réunis en collège sur le modèle d'autres secteurs de formation en médecine et santé. Ces collègues ont organisé plusieurs rencontres sur la question et ont déjà publié un manuel dont une version numérique sera disponible très bientôt ${ }^{32}$ (Visier, 2011).

C'est en s'appuyant sur les travaux qui ont accompagné les évolutions de ces deux contextes de formation, que Muriel Guedj propose une réflexion sur la place de l'épistémologie et de l'histoire des sciences et techniques (EHST) (comprises comme un cas paradigmatique de sciences humaines et sociales) dans la formation des enseignants de sciences expérimentales. Cette réflexion la conduit à mettre en avant les recherches sur le contexte changeant de la formation des enseignants en général, et plus spécifiquement sur la caractérisation fine de la professionnalité des enseignants de sciences expérimentales. Suite aux évolutions institutionnelles dont on a rappelé plus haut les grandes lignes (§0.3), on sait qu'il existe depuis 2007 pour la formation des enseignants français un « référentiel de compétences ${ }^{33}$ : d'abord inspiré en 2007 par des référentiels francophones (canadiens), il a subi récemment une première révision ${ }^{34}$. Les débats qui ont accompagné cette révision ont mis en valeur le besoin d'une réflexion continue sur les évolutions rapides de la professionnalité enseignante, dans le contexte changeant évoqué ici par l'auteure en reprenant les termes d'un rapport de l'OCDE. Il reste certainement, comme le suggère la proposition de réflexion comparatiste de M. Guedj, à garder vivante cette réflexion et à la spécifier pour ce qui concerne celle des enseignants de sciences et techniques, en la mettant de surcroît en perspective avec les évolutions concomitantes d'autres champs professionnels.

Notons ici que la contribution de G. Aiguier et C. Didier sur la formation à l'éthique dans les études médicales et d'ingénieurs (02003), même si nous avons choisi de la regarder comme une étude locale pour des raisons qui seront expliquées plus loin (\$2), pose semblablement les bases d'une comparaison entre deux domaines de formation professionnelle, à propos d'un objet de formation dont l'identité est bien plus problématique que l'EHST, à savoir l'éthique. Pour eux, l'éthique s'apparente à « une compétence que les professionnels doivent développer pour revisiter les fondements, les finalités et les moyens de leur agir et ainsi s'engager dans une pratique professionnelle plus critique, plus réflexive et plus responsable » et ainsi permettre à de futurs professionnels de se situer dans un champ complexe et en mutation. Autrement dit, dans une situation (française) où la question peine encore à trouver une définition claire, de manière similaire à ce que propose Muriel Guedj, c'est une investigation préalable sur la place de l'éthique dans la définition de la professionnalité des médecins qui oriente une réflexion prospective sur la place possible de la formation à l'éthique dans les cursus d'ingénieur.

\subsection{Trois approches pour la formation en sciences humaines et sociales dans les écoles d'ingénieurs}

Les trois premières contributions affrontent un objet commun, à savoir la remarquable diversité de l'inscription des sciences humaines dans la formation des ingénieurs français et européens, mais avec des approches très différentes. C. Roby et $\mathrm{M}$. Cotte tentent en effet de rendre plus lisible la diversité de l'inscription des sciences humaines, ou de ce qui en tient lieu, dans les formations d'ingénieurs en France $^{35}$. La première s'inscrit dans la continuité de travaux récents, quoique peu nombreux encore, en sciences de l'éducation : elle s'appuie plus particulièrement sur la sociologie du curriculum pour

\footnotetext{
32 Lors du colloque associé à ce recueil, cette réflexion collective déjà vieille de deux décennies a fait l'objet d'un exposé récapitulatif par Christian Bonah, le premier président du collège des enseignants de sciences humaines et sociales en médecine et santé, et l'un des co-éditeurs du Manuel. Voir http://shst2013-upec.sciencesconf .org/12639 (consulté 19.8.14).

33 Il concerne en général les métiers de l'enseignement et de l'éducation.

34 En 2013, à l'occasion de la loi sur la refondation de l'école.

35 Elles sont envisagées dans les deux cas de manière quasi exhaustive.
} 
proposer, à partir d'un examen quasi exhaustif des curricula des écoles d'ingénieurs contemporaines, une catégorisation originale des différentes manières dont les sciences humaines s'y inscrivent. Cette approche est complétée par une étude de cas succincte qui met en œuvre, sur le cas de formations agricoles, la notion intégrative de culture technique proposée par des collègues canadiens, notion qui prolonge d'une part les apports de la sociologie du curriculum et rejoint à certains égards celle de Cotte.

Ce dernier propose en effet d'emblée une lecture historique originale ${ }^{36}$ de l'inscription d'une dimension «humaniste» au sens large, en tâchant de restituer les racines, déjà anciennes et bien attestées dès le 18ème siècle, d'une composante de formation «non strictement » scientifique et technique dans les cursus d'ingénieurs, bien avant que n'existent des spécialistes et enseignants de « sciences humaines et sociales » au sens académique du terme. Cette synthèse le conduit en particulier à resituer, sur des bases déjà installées en Europe à la fin du 18ème siècle, la construction au cours du 19ème siècle de deux profils d'écoles, les unes plutôt généralistes les autres plus spécialisées, puis à appréhender la complexification de ces profils fondamentaux au cours du 20ème siècle, où des mariages originaux avec des structures universitaires se sont mis en place.

Ces deux approches différentes conduisent ainsi à une relative divergence dans l'approche de l'objet « sciences humaines et sociales »: l'une, récente, comprend sous ce nom, de manière positive et en partie militante, un ensemble de champs académiques institués, susceptibles d'enrichir et de rénover la formation des ingénieurs. La catégorisation proposée offre un spectre de possibilités qui vont de l'intégration faible ou tacite des sciences humaines et sociales à la formation à une intégration forte et revendiquée ; elle reprend en outre les catégories contemporaines instituées par le conseil national des universités pour désigner les champs disciplinaires dont on étudie la plus ou moins grande intégration. Le point de vue historien est naturellement conduit à questionner la définition d'un objet pérenne sur deux siècles, et propose ainsi une définition négative des composantes « humanistes » de la formation, comme « tout ce qui ne relève pas strictement d'une formation scientifique et technique ${ }^{37}$. Le premier point de vue s'avère du coup naturellement adapté à un point de vue militant qui conduit à la généralisation d'une conception fortement «intégrée » des sciences humaines et sociales à la formation des ingénieurs ${ }^{38}$. Le second point de vue, par contre, relativise fondamentalement la présence des sciences humaines et sociales, de création récente, dans la formation des ingénieurs. Elle l'inscrit non seulement dans l'histoire longue des espaces «d'humanités » qui les ont précédées, mais restitue aussi le jeu complexe des contraintes institutionnelles et historiques qui gouvernent en grande partie cette inscription, suivant les secteurs professionnels, le type d'école considéré et sa vocation. De ce point de vue, cette approche rejoint les approches moins militantes également illustrées dans ce volume par plusieurs contributions ${ }^{39}$, qui tendent à restituer la complexité des logiques historiques et institutionnelles permettant de bâtir des compromis et se situant ainsi dans un empan plus large que les possibilités répertoriées par C. Roby.

Ces deux points de vue, sociologique et historien, peuvent bien-sûr être vus comme complémentaires, ouvrant la voie vers différentes pistes, par exemple une sociologie historique qui a

\footnotetext{
36 Nous avions au départ convié M. Cotte à proposer en conférence plénière un « simple » état des lieux sur les enseignements de sciences humaines en écoles d'ingénieurs (http: //shst2013-upec.sciencesconf .org/13452 consulté 19.8.14). Devant le résultat foisonnant et manifestement novateur de cette contribution, nous lui avons demandé de tourner ses réflexions en une contribution à ce volume, ce qui nous a valu deux articles complémentaires, dont la synthèse ici présentée ! Qu'il soit à nouveau remercié de ces éclaircissements originaux et très suggestifs.

37 Cette définition négative rejoint à certains égards la définition également négative de l'interdisciplinarité proposée par Godfroy, comme nous le verrons plus loin.

38 Ce point de vue est bien illustré dans le même volume par d'autres contributions, comme celle de G. Quiquerez (05003), G. Carnino (05002) ou C. Lenay et ses collègues (05001). Concernant l'UTBM, on pourra également lire la seconde partie de la seconde contribution de M. Cotte, dans la section suivante (02001).

${ }^{39}$ C'est le cas des approches de Michel Cotte à nouveau $(02001, \S 1)$ pour le Polytech Nantes, N. Hourcade (02002), ou C. Colmelerre (03001) pour l'Ecole Centrale.
} 
bien été illustrée, lors du colloque, par l'étude de cas défendue par Antoine Derouet ${ }^{40}$. La notion de culture technique mise en valeur par $\mathrm{C}$. Roby en complément de son approche sociologique s'avère également adéquate pour comprendre des logiques historiques et génétiques, suivant les secteurs qu'on cherche ainsi à caractériser. On peut également consulter, dans la même orientation, les travaux d'André Grelon et de ses collègues (Grelon 2007).

Par ailleurs cette double approche historique et prospective doit être mise en regard de la troisième approche originale proposée par A.-S. Godfroy. Partant d'un autre questionnement initial concernant la question des facteurs d'attractivité des formations d'ingénieurs en Europe, notamment vis-à-vis des femmes, et des résultats d'un projet de recherche européen dédié à ces questions (le projet HELENA), Godfroy propose une sorte de retractatio méthodologique visant à critiquer en profondeur les critères de comparaison et de définition adoptés pour le projet de recherche comparatif en question. D'une manière similaire au recours que fait Roby à la notion subsumante de culture technique, Godfroy fait appel à une notion d'interdisciplinarité qui d'une part recouvre en partie la problématique des sciences humaines (d'emblée classées comme « interdisciplinaires » et qui de l'autre rappelle la définition négative proposée par M. Cotte pour les besoins de son approche historique : est «interdisciplinaire » tout ce qui ne relève pas strictement d'une formation scientifique et technique. En questionnant, pour les réviser et les perfectionner, les faiblesses de cette première approche, Godfroy est conduite à définir sur un mode prospectif un certain nombre de grandes questions permettant d'asseoir des approches mieux définies de l'objet initial de recherche.

\subsection{Du comparatisme à la définition d'approches prospectives}

Parmi les approches retenues, Godfroy retient l'approche socio-historique dont les contributions de Cotte et Roby montrent l'importance, comme on l'a vu plus haut. Elle retient par ailleurs, en l'inscrivant dans la perspective contemporaine du renouvellement $\mathrm{du}$ 《 chaos des disciplines », des questions épistémologiques fines sur la définition et l'articulation des disciplines entre elles et dans un tout cohérent. Mais elle souligne surtout une leçon majeure tirée du projet de recherche qui a servi de départ à ces réflexions, à savoir la nécessité fortement ressentie et marquée d'adapter l'ingénierie des formations aux parcours désormais complexes des étudiants ou des professionnels. Dans cette lecture, la place des sciences humaines et sociales pourrait être lue comme un facteur de choix dans la stratégie complexe de construction et d'enrichissement par les étudiants de leurs parcours de formation, et plus seulement comme une composante d'un plan de formation uniformément applicable à tous les étudiants. Cette question renvoie à l'existence de systèmes d'options, comme on le verra à travers plusieurs exemples dans la partie suivante ${ }^{41}$. A.-S. Godfroy souligne également (et corrélativement au point précédent) que les questions d'ingénierie pédagogique, de façons d'enseigner, doivent être un des axes de recherche à approfondir afin de définir les termes d'une enquête plus précise : plusieurs contributions à ce volume, notamment celles de la 3ème section (Dispositifs pédagogiques), abordent cette question.

Comme on l'a vu plus haut $(\$ 1.1)$, cette partie se conclut par un autre article prospectif, celui de M. Guedj sur la transposition possible des questionnements valables pour la formation des ingénieurs et médecins, à la formation des enseignants. On peut lire cette contribution à un niveau général comme l'une des voies de transposition de questionnements d'un domaine de formation professionnelle à l'autre, parmi d'autres qui resteraient à approfondir. On peut se demander notamment si le fait de subordonner le positionnement des sciences humaines et sociales au questionnement sur la professionnalité visée, ne revient pas de facto à privilégier une logique « adaptative » contre une

\footnotetext{
40 http://shst2013-upec.sciencesconf .org/10018 consulté 19.8.14. M. Derouet n'a pas souhaité contribuer au volume, mais on peut lire (Derouet 2013).

${ }^{41}$ Notamment dans les contributions de M. Cotte (02001, partie 1) et N. Hourcade (02002) : la question des systèmes d'options s'avère cruciale pour le développement des enseignements de sciences humaines et sociales dans les deux contextes évoqués.
} 
logique « émancipatrice » qui devrait permettre de penser, par exemple, l'usage désormais uniforme de référentiels de compétences dans la définition de cette professionnalité ${ }^{42}$. On retrouve ici, sans étonnement, l'une des questions fondamentales qui traversent un grand nombre des contributions de ce volume ${ }^{43}$.

Mais on peut aussi lire la contribution de M. Guedj pour ce qu'elle propose finalement, à savoir un exemple concret de déclinaison de cet effort de transposition, en l'occurrence par le développement d'un programme de recherche sur la place de l'énergie dans l'enseignement et la formation en sciences expérimentales. De même, l'article de C. Roby propose un exemple tiré de l'exemple des études d'ingénierie dans le domaine agricole, et celui de $\mathrm{M}$. Cotte peut être vu comme une introduction à une (double) étude de cas qui fait l'objet d'un second article du même volume (02001). En général, cette partie comparatiste trouve un prolongement et une illustration utiles par les études de cas contenues dans les deux parties suivantes.

\section{Influence du contexte local}

\subsection{Présentation générale : quatre études de cas illustrant des collaborations locales}

Sur la base de l'analyse comparative et prospective menée dans les articles de la première section de l'ouvrage, il nous a été possible de rassembler dans cette seconde section cinq études de cas très éclairantes qui ont toutes en commun d'illustrer l'influence du contexte local (enseignants impliqués et responsables administratifs) sur la mise en place d'enseignements de sciences humaines dans les formations professionnalisantes de futurs scientifiques, ainsi que le caractère hautement collaboratif de cette mise en place.

Les deux premières contributions, de Michel Cotte (de nouveau 02001) et Nicolas Hourcade (02002), sont des études de cas concrètes de mise en place par des équipes pluridisciplinaires de formations sciences humaines et sociales dans trois contextes institutionnels : Polytech Nantes et l'UTBM pour le premier, l'école Centrale de Lyon pour le second. Ces études montrent non seulement que le paramètre institutionnel est une variable essentielle à prendre en compte pour saisir les conditions de développement d'une telle offre, mais encore qu'il a des conséquences sur la nature même des choix d'enseignements en sciences humaines et sociales et les recherches associées.

La troisième évoque le travail de concertation conduit dans une même université pluridisciplinaire (l'université Catholique de Lille) autour des enseignements d'éthique dans les formations médicales d'une part, et les formations en école d'ingénieurs de l'autre (G. Aiguier et C. Didier 02003). La dernière contribution décrit et problématise à l'échelle de l'université Paris-Est Créteil une collaboration autour la mise en place d'enseignements d'Epistémologie et histoire des sciences et des techniques (EHST) pour la formation des enseignants en sciences et techniques (02004). Les deux contributions procèdent de nouveau à une recontextualisation institutionnelle et professionnelle, et permettent de mettre en perspective les effets de ce contexte sur la proposition et la mise-en-œuvre de la formation; on verra là encore que la question de la définition des champs de recherche associés à cet effort de formation se pose très fortement.

\subsection{Les expériences comparées de Polytech Nantes, de I'Université de Technologie de Belfort-Montbéliard et de l'Ecole Centrale de Lyon}

La contribution de Michel Cotte est le complément direct de son étude historique globale dont on a résumé plus haut le contenu (01002). En se fondant sur sa très riche expérience de directeur d'un

\footnotetext{
42 Voir par exemple les travaux critiques de J. Le Goff sur la question, par exemple (Le Goff 1996).

43 Voir notamment les articles de Colmelerre (03001), Gaglio (03002) et Carnino (05002) qui toutes trois abordent cette question au sujet du statut de la sociologie.
} 
département de sciences humaines et sociales en écoles d'ingénieurs, expérience conduite dans deux contextes institutionnels très différents, l'auteur propose principalement, dans le cas de Polytech Nantes, une analyse détaillée du processus lent et complexe qui a permis l'établissement d'une offre de cours en sciences humaines. Cette offre s'est construite avec l'Institut de l'homme et de la technologie (IHT) dans le contexte de la fusion d'un ensemble de plusieurs écoles de taille modeste au sein de l'université de Nantes où ces écoles sont devenues départements de Polytech. Tout l'intérêt de ce retour réflexif sur les activités du département transversal «Homme, Entreprise, Société » (HES) est de montrer comment le changement d'échelle, l'intégration à l'université et une politique volontariste issue du soutien de la direction (dans le cadre du renforcement de la politique globale de l'école) ainsi que des négociations patientes des membres du département, ont permis la constitution d'une offre transversale de cours de sciences humaines dans un contexte où elle n'allait pas de soi, en raison des habitudes et traditions propres aux départements et aux difficultés propres à l'émergence d'une culture d'établissement.

Comme M. Cotte l'indique dans son premier article, cette expérience est d'autant plus intéressante et instructive qu'elle se situe dans le contexte d'une école « à dominante spécialiste » dont l'enjeu principal n'est donc pas de former des ingénieurs 'généralistes' ni d'afficher une forte composante « sociétale » : on touche donc là à un milieu peu visible et exploré, mais dont l'existence et le développement sont bien réels. Cette perspective visant à explorer la «face obscure » des sciences humaines et sociales en école d'ingénieurs rejoint donc l'approche revendiquée par Bernard, de Montgolfier et Roux pour le développement des sciences humaines et sociales dans des contextes universitaires, comme on le verra plus loin.

Michel Cotte propose ensuite un contrepoint, en comparant cette expérience à celle de la direction des études dans le département des « humanités » d'une université de technologie (celle de BelfortMontbéliard, l'UTBM) où la construction d'une offre en sciences humaines et sociales est infiniment moins problématique car inscrite structurellement dans une politique d'école et une conception globale qui donnait, dès le départ, une place très importante à ce type d'enseignements ${ }^{44}$. Les moyens alloués à leur développement, et la force de proposition des équipes du département des « humanités », sont donc nettement plus importants dans ce contexte que dans le précédent. La comparaison des deux expériences conduit enfin M. Cotte à des conclusions plus générales qui constituent en soi une véritable question de recherche, sur la nature des sciences humaines et sociales en écoles d'ingénieur, sur lesquelles nous reviendrons plus loin.

Dans le même cadre de la formation des ingénieurs, mais cette fois-ci dans le contexte d'une école qui revendique de manière marquée une vocation « généraliste » (formation d'ingénieurs « managers »), Nicolas Hourcade nous propose une analyse locale à l'Ecole Centrale de Lyon (ECL) des différents types de formation en sciences humaines et sociales sur l'ensemble du cursus de la formation de l'ingénieur. Son objectif, très semblable à celui que suit M. Cotte pour le Polytech Nantes, est de nous montrer comment ces enseignements sont apparus historiquement, quel est le rôle des différents profils des enseignants impliqués dans ces formations, et enfin quelle est (à nouveau) la place non négligeable tenue par la politique active de la direction dans le soutien à l'existence même de ces formations, dont le développement doit être patiemment négocié, comme dans le Polytech, avec le périmètre des enseignements « $\mathrm{SPI}^{45} »$. Un point original que souligne Hourcade, et d'autant plus intéressant qu'on pourra le mettre en regard des expériences de l'UTBM d'un côté, et de Polytech de l'autre, est la place très particulière tenue par le développement de la recherche dans ce contexte : au départ, le développement des sciences humaines et sociales était associé à la création volontariste d'un laboratoire interdisciplinaire associant l'économie, la sociologie, les science du langage, l'informatique et les

\footnotetext{
44 Quant à la conception en question, elle est décrite plus en détail dans la contribution de Charles Lenay et de ses collaborateurs (05001) dans leur contribution du même volume. On pourra également lire la troisième partie de la contribution de G. Carnino (05002) pour le cas de l'université de technologie de Compiègne (UTC).

45 Sciences Pour l'Ingénieur.
} 
sciences de l'information, mais cette politique a échoué sous sa forme primitive et le développement des sciences humaines et sociales s'est en réalité appuyé pour l'essentiel sur celui des enseignements, la question de la formation à la recherche se posant désormais d'une manière toute différente. Le caractère «généraliste » de l'ECL ne doit donc pas faire écran : si l'ambition affichée est celle d'une université de technologie à certains égards, la réalité du travail collaboratif la rapproche d'écoles spécialisées, elle se situe dans une sorte d'intéressant mi-chemin entre les deux expériences décrites et analysées par Michel Cotte.

\subsection{L'exploration des potentialités d'une expérience collaborative dans l'université Catholique de Lille et à l'université Paris-Est-Créteil}

On a vu plus haut $(\$ 1.1)$ que la troisième contribution de cette section pose en préalable le problème de la définition d'un enseignement d'éthique dans les cursus de formations de personnels de santé et d'ingénieurs. Par contraste avec la quasi absence ou invisibilité de cette thématique de formation, notamment dans les formations d'ingénieurs françaises ${ }^{46}, \mathrm{G}$. Aiguier et $\mathrm{C}$. Didier évoquent la structure originale propre à l'université Catholique de Lille, le département d'Ethique de l'Institut Catholique de Lille qui leur permet de comparer leurs expériences de formation en sciences humaines et sociales focalisées sur le thème de l'éthique dans deux contextes différents : celle des études médicales, et celles des écoles d'ingénieurs. On a vu plus haut (\$1.2) que leur étude s'ancrait dans une réflexion sur la nouvelle définition de la professionnalité, qu'il s'agisse des médecins ou des ingénieurs. L'article a en outre pour objectif de mettre en exergue les deux types de formation avec leurs différences, tant dans leur origine historique, que dans leur intégration au sein des curriculums de formation et dans le champ professionnel. Si les auteurs donnent relativement peu de détails sur la structure institutionnelle transversale qui permet ce travail, on en voit cependant les premiers effets : l'un des points communs mis en exergue dans l'article concerne en effet la posture pédagogique de l'enseignant dans ce type de formation qui doit pour eux varier en fonction des étapes de la formation, l'apprentissage étant vu selon une perspective socioconstructiviste ${ }^{47}$. Pour compléter cet article nous invitons le lecteur à comparer cette approche avec l'approche défendue par G. Quiquerez dans sa contribution, dans laquelle il renvoie implicitement à une autre expérience d'enseignement à l'Ecole Centrale de Marseille, ainsi qu'avec l'approche pédagogique proposée par Cynthia Colmellere en école d'ingénieurs (03001 et $\$ 3$ ci-dessous). Enfin, on pourra rapprocher ces réflexions autour des enseignements d'éthique des contributions publiées dans des actes du colloque sur "la formation des scientifiques à l'éthique » qui s'est tenu le 15 février 2013 (Byk 2014) (8) $^{48}$

La dernière contribution est due à trois d'entre nous. Focalisée sur l'université Paris-Est-Créteil (UPEC) qui a accueilli le colloque, elle fait fortement écho avec les remarques présentes dans les trois autres contributions concernant le caractère éminemment collaboratif de la mise en place d'enseignements en sciences humaines et sociales dans un contexte professionnel contraint. Elle interroge plus spécifiquement, en proposant de les inscrire dans le modèle des communautés de pratique proposé au départ par Etienne Wenger, les relations collaboratives qui peuvent se nouer dans le cadre local d'une université entre les différents enseignants impliqués dans des formations en sciences humaines et sociales. Il s'agit ainsi de décrire une expérience locale, focalisée en l'occurrence autour

\footnotetext{
46 C'est ce que confirment encore les remarques de Michel Cotte dans sa contribution sur les cours de Polytech Nantes : à la fin de l'année 90, les incitations de la CTI à introduire de l'éthique dans les cursus se traduisaient par une incompréhension générale sur la nature précise de cette demande.

47 On rapprochera cette approche de la description donnée par Colmellerre de son rôle au cours des séances décrites dans sa contribution (section suivante, Dispositifs pédagogiques, et contribution 03001).

${ }^{48}$ Les contributions sont audibles sur le site du ministère des affaires étrangères (http://www.diplomatie.gouv.fr/ $\mathrm{fr} /$ politique-etrangere-de-la-france/onu/la-france-et-l-unesco/commission-francaise-pour-l-unesco/ sciences-21752/actualites-21757/article/colloque-formation-des-104747, consulté le 06.10.14).
} 
d'enseignements d'histoire des sciences pour de futurs enseignants de mathématiques, de sciences expérimentales ou de disciplines technologiques, et de théoriser à travers cet exemple la richesse et les limites d'une pratique collaborative dans de tels contextes où le développement des sciences humaines et sociales est précaire, au sens où il n'est pas soutenu par un contexte institutionnel fort. Bernard et al. analysent ainsi spécifiquement le développement de la pratique collaborative d'une micro-communauté d'enseignants, en empruntant aux « communautés de pratique » d'Etienne Wenger le cadre théorique qui permet de décrire des expériences de mutualisation et d'en comprendre les conditions d'émergence.

\subsection{Conclusions prospectives : la redéfinition des sciences humaines et sociales en contexte professionnalisant, et la fédération locale de communautés d'enseignants-chercheurs autour de projets communs}

Les quatre contributions de cette section présentent un point commun saisissant : elles insistent toutes d'une manière ou d'une autre sur l'importance de l'articulation entre un projet collaboratif d'enseignement et la définition de champs de recherche compatibles avec cette activité.

Que toutes les contributions insistent, chacune à sa manière, sur la nécessité d'une collaboration forte entre les enseignants impliqués dans la mise en place de formations en sciences humaines, dans chacun des contextes étudiés, est une évidence. Cette nécessité est non moins clairement à rapporter au contexte contraint des milieux professionnalisants qui oblige par nature à négocier la position de ces enseignements vis-à-vis des enseignements « dominants » du cursus, comme l'explique Michel Cotte dans sa conclusion au sujet des travaux qu'il analyse, mais la remarque peut être généralisée. Il faut donc s'entendre et se coordonner, pour définir une offre d'enseignements à la fois cohérente, souple et adaptée. Dans la dernière contribution, c'est précisément ce fonctionnement souple et évolutif qu'on propose de penser à l'aune de la théorie des communautés de pratique.

Le point plus subtil est lié à la définition d'une politique de recherche fédérative, voire à la définition de nouveaux champs de recherche en sciences humaines et sociales. Le cas «simple », si l'on peut dire, est celui que représentent les universités de technologie, structurées dès leur fondation pour articuler des projets interdisciplinaires entre les sciences pour l'ingénieur et les sciences humaines et sociales, et qui peuvent dans certains cas se prévaloir de structures fortes et adossées à des laboratoires, comme le laboratoire RECITS à l'UTBM, ou des structures fédératives de recherche comme celles que définissent la contribution de C. Lenay et ses collègues, dans ce volume (05001). Dans ce cas, la même contribution montre que cet effort de fédération passe par un essai de redéfinition de ce que signifie précisément une recherche en sciences humaines profondément intégrée à l'enseignement et la recherche technologiques ${ }^{49}$.

Les autres cas, à la fois moins visibles et plus précaires du point de vue du regroupement des enseignants dans des entités fortes associées à un programme de recherche, posent en réalité des problèmes voisins. L'analyse de $\mathrm{N}$. Hourcade commence, de manière remarquable, par le récit d'un échec à fédérer une équipe d'enseignants autour d'une problématique interdisciplinaire de recherche, et se conclut par une analyse nuancée de la situation actuelle, où le lien entre enseignement et recherche est résolu de manière différente, sous forme d'arrangements individuels, au risque d'une certaine précarité de l'équipe. L'analyse d'A. Bernard et alii pointe également en conclusion le rôle fédérateur du projet de recherche évolutif dans la construction des enseignements qu'ils rapportent. Enfin l'analyse de G. Aiguier et C. Didier montre à l'évidence que la question de la conception d'une «posture commune » d'enseignement n'est ni dissociée ni dissociable d'un effort de recherche dans ce domaine - c'est ce dont témoigne la journée d'études organisée récemment sur ce sujet dans le même contexte.

\footnotetext{
${ }^{49}$ La contribution de G. Carnino peut être vue, à cet égard, comme un essai similaire pour définir un nouveau champ de recherche - l'épistémotechnique - articulé fondamentalement à la conception des enseignements rénovés.
} 
Enfin nous attirons l'attention sur une des conclusions fortes de l'article de Michel Cotte, au sujet de la nature exacte des sciences humaines et sociales quand elles se développent dans un contexte professionnalisant, comme celui des écoles d'ingénieurs. S'agit-il d'une sorte d'extension des sciences humaines et sociales « académiques » vers des objets nouveaux, ou bien de nouvelles sortes de sciences humaines et sociales qui n'ont de sens plein que dans ce contexte, à l'image de l'ergonomie ou de la sociologie des organisations ? L'exemple de ce champ en émergence qu'est "l'éthique pour ingénieurs », en tant que domaine de formation et de recherche en voie de légitimation, en donne un exemple éclatant et bien illustré par la contribution de G. Aiguier ou C. Didier. Michel Cotte signale à juste titre l'importance de ce problème, et la nécessité d'un faire une question de recherche ; nous signalons à notre tour qu'elle est probablement générale. Pour le domaine de la formation des enseignants et des médiateurs culturels, c'est ce qu'illustre dans ce même volume la contribution de $\mathrm{S}$. Laubé et G. Chambon (04002).

\section{Dispositifs pédagogiques}

L'introduction d'enseignements de sciences humaines dans les cursus du supérieur, au-delà de bousculer des habitudes propres à tel ou tel contexte, pose le problème très concret de leur mise en œuvre. Les différentes contributions regroupées dans cette troisième partie nous proposent l'analyse de dispositifs nouvellement mis en œuvre -et qui ont d'une façon ou d'une autre renouvelé les méthodes d'enseignement.

Ainsi, pour des futurs ingénieurs en deuxième année à l'École Centrale de Paris, Cynthia Colmellere (03001) présente un dispositif pédagogique autour d'un exercice de débat public sur l'implantation d'un réacteur nucléaire dans le cadre d'un cours de sociologie. Ce dispositif interroge à la fois les étudiants sur les dimensions éthiques de leurs pratiques de travail et le rôle qu'ils auront à jouer dans la société et les enseignants pour suggérer une démarche à laquelle ils ont rarement été formés, le débat. Il questionne également la formation des ingénieurs aux dimensions sociales et politiques de la technique. Colmellere expose les différentes étapes d'une familiarisation des étudiants avec un dossier qu'ils n'ont jusqu'alors envisagé que sous un angle scientifique. L'enjeu fort est de les faire passer «d'un jugement à l'interrogation, puis, in fine à une problématisation de la question nucléaire » tout en les questionnant sur les conditions d'un débat démocratique.

Cette auteure montre bien comment le débat peut être utilisé en classe à un niveau universitaire pour sensibiliser les élèves à des questions qui ne sont pas mises en avant dans leurs cours de physique car elles concernent des questions dites socialement vives. Cela permet de mener une réflexion épistémologique telle que celle qui est analysée par Legardez et Simonneaux $(2006,2011)$ ou Albe (2009) autour de questions socialement vives (Simonneaux, 2003) ${ }^{50}$. De nombreux auteurs ont montré l'intérêt du débat pour conduire les élèves de l'élémentaire jusqu'au supérieur à problématiser (Orange 2012; Lhoste 2008). Ils se réfèrent à la démarche d'investigation pour caractériser ces activités menées dans des enseignements scientifiques traditionnels.

Toujours dans le cadre d'un enseignement de sociologie pour des élèves-ingénieurs, Gérald Gaglio (03002) présente des enseignements réalisés à l’Université de Technologie de Troyes (UTT). Son objectif est de mettre en évidence différents statuts de la sociologie : d'une part, la sociologie est enseignée pour préparer les étudiants au monde du travail via une socialisation, et d'autre part, il convient d'éviter de présenter la sociologie seulement selon des conceptions utilitaristes. Une expérience pédagogique particulière est mise en œuvre dans le cadre d'un module de «Sociologiedes organisations

\footnotetext{
50 Ces QSV sont définies comme triplement socialement vives par Simonneaux (2003) (a) parce qu'elles suscitent des débats dans la production des savoirs savants de référence; (b) parce qu'elles sont prégnantes dans l'environnement social et médiatique, et que les acteurs de la situation didactique (élèves et enseignants) ne peuvent y échapper; enfin (c) parce qu'en classe, les enseignants se sentent souvent démunis pour les aborder.
} 
pour l'ingénieur en système d'information » consistant à analyser et problématiser une expérience de stage de six mois que tous les élèves-ingénieurs ont vécue, notamment à la lumière de la « sociologie orientée ». L'article décrit le dispositif mis en place pour provoquer un renversement du regard des futurs ingénieurs sur leur expérience professionnelle. Ainsi, il tente de les faire passer d'une vision opérationnelle à une vision sociologique. Il discute différents exemples qui mettent en relief la portée et les limites de cette démarche pédagogique dans ce contexte de formation d'ingénieurs, notamment sur le thème de la « résistance au changement ». Son article complète celui de Cynthia Colmellere en proposant une autre piste pédagogique conduisant les futurs professionnels vers une réflexion épistémologique.

Sur le plan des débats au sujet de l'utilité et de la fonction de la sociologie en formation, cet article est à mettre en regard des prises de positions qui montrent la vitalité de ce débat parmi les sociologues, par exemple avec Lahire (2004), Dubet (2011) ou bien, dans l'article de Colmellere, la partie introductive dans laquelle elle situe son approche par rapport à d'autres.

Pour un tout autre public, dans le cadre de la mise en place du «Plan réussite en licence ${ }^{51}$ au sein de l'université Paris-Diderot, le département Sciences de la Nature et de la Vie a inscrit une série de modules déployés autour de la préprofessionnalisation. Hervé Duchauffour (03003) nous expose les réflexions menées autour de la mise en place d'enseignements réalisés en co-intervention entre enseignants de sciences humaines et enseignants de biologie. Il nous montre comment cette rencontre est vécue au départ comme une mise en danger par les biologistes préoccupés par la perte de «la maîtrise de leur enseignement » et donc par le risque de «fragiliser leur stature de spécialistes disciplinaires ». Il montre également la volonté des enseignants scientifiques de se mettre à l'épreuve dans des situations de cours peu habituelles. Quelle que soit leur ancienneté, l'auteur pointe leur capacité à prendre de la distance par rapport à des pratiques d'enseignement purement liées à la discipline. Les modalités de travail utilisées permettent une centration sur l'apprenant au travers d'ateliers d'échanges, de recherches documentaires, de jeux de rôle ou d'exposés. Après plusieurs années de pratiques, la plupart des enseignants témoignent de changements dans leurs représentations de leur activité d'enseignant et présentent ce qui a changé pour eux. Cet article permet de poursuivre une réflexion déjà engagée sur les pratiques de préprofessionnalisation en s'attachant à des profils d'enseignants particuliers.

Enfin Jérôme Fatet (03004) propose une analyse qui concerne spécifiquement le contexte de la formation des enseignants et met l'accent sur la réflexion sur le sens de la pratique scientifique et la construction d'un savoir scientifique. Il ne néglige pas non plus les contraintes techniques liées à un enseignement de sciences physiques.

A l'université du Limoges, au sein de l'ESPE du Limousin, J. Fatet a en effet développé sur plusieurs années des activités de réplications d'expériences historiques, permettant aux étudiants en master MEEF de se familiariser avec les manipulations d'anciens appareils scientifiques de mesures. Cette démarche a été initiée suite à un bilan mitigé de l'impact d'enseignements d'EHST traditionnels, en ce qui concerne l'évolution des conceptions des étudiants sur la nature des sciences. A partir de ce constat, Jérôme Fatet a developpé une approche expérimentale de certaines questions centrales de l'épistémologie des sciences. Il a proposé une série de réplications d'expériences historiques permettant de replacer les étudiants dans des contextes épistémologiques différents de ceux qu'ils connaissaient. Un de ces enjeux était de suggérer à ces futurs enseignants de mettre en place ce type d'activités dans leurs établissements du second degré. Tout au long des différentes étapes du travail de réplication d'expériences historiques, l'accent est mis sur le rôle de l'expérience dans l'élaboration et la transmission des savoirs, le statut des instruments pour l'exploration expérimentale ainsi que la signification du résultat numérique lors de l'exploitation d'une expérience.

Plusieurs universités ont valorisé l'utilisation de dispositifs expérimentaux historiques avec les étudiants. A Oldenburg, Dietmar et Höttecke (2000) ont montré l'efficacité de telles méthodes sur

51 Il s'agit d'un plan annoncé par la ministre de l'enseignement supérieur et de la recherche Valérie Pécresse en décembre 2007. 
l'apprentissage, et Heering (2007) a élaboré des formes pédagogiques de réplication. A Cambridge, Chang (2010) a pointé une amélioration de la compréhension de la nature de la science par les étudiants. On peut y voir des prolongements des méthodologies d'apprentissage développées au niveau européen, « learning by doing » (Heering, Wittje 2011) et « hands-on/minds-on activities » (Rocard, 2007). On les retrouve dans d'autres champs d'apprentissage tels la muséologie ${ }^{52}$.

Si l'on porte un regard sur l'ensemble de ces dispositifs, force est de constater que ce qui fait leur attrait principal est un rapport différent aux étudiants qui n'apparaissent plus comme des creusets qu'il faut remplir de connaissances mais comme des personnalités humaines, capable d'autonomie et possédants différentes compétences transversales à développer. La perception des étudiants est prise en compte dans ces démarches innovantes.

L'autre élément qui revient (sous forme de constat ou de souhait) est l'intérêt de travailler à plusieurs enseignants, aussi bien pour croiser les regards que l'on porte et enrichir l'apport aux étudiants, que pour découvrir d'autres démarches d'enseignement ou tout simplement être rassuré. On rejoint ici une des conclusions majeures de notre analyse des contributions de la précédente section (Sect. 3).

\section{Formation et recherche}

\subsection{Le développement concomitant d'une activité de recherche et de formation : un paradigme utile pour analyser les trois contributions de cette partie}

Le binôme " recherche et formation », comme le rappellent Françoise Clerc et Jean-Marie Barbier dans un entretien publié dans la revue du même nom (Barbier et Clerc 2008), est ambigu dans son couplage comme dans l'identité de chacun des termes proposés. Compris dans le sens le plus large, où tous les liens possibles entre la recherche académique (en l'occurrence en sciences humaines et sociales) et la formation (ici les formations professionnalisantes en sciences et techniques), sont envisageables, à peu près toutes les contributions de ce volume sont concernées. En effet, on a vu que même les contributions se présentant comme des témoignages circonstanciés, comme ceux qui ont été regroupés dans la troisième section, renvoient directement à la question de la fédération des enseignants concernés autour de projets de recherche (cf. §2.4). S'il faut donc faire, avec J.M. Barbier, le constat raisonnable de «la relative diversité de cultures de pensée et d'action autour des liens entre recherche et formation » suivant les objets d'étude et les champs concernés, on peut au moins distinguer deux paradigmes, un paradigme « applicatif » où la production de connaissances scientifiques nouvelles et leur rapport à la formation est compris comme une forme d'application, et un autre plus récent, et mieux adapté globalement aux tentatives contemporaines d'accorder formation à la recherche et formation professionnelle en général, « qui lie action, production de savoirs et construction des sujets humains ». Quoiqu'il en soit de cette dichotomie peut-être simplificatrice à certains égards ${ }^{53}$, il n'en reste pas moins que le second paradigme présente au moins deux grands avantages. D'une part il est reconnaissable dans les évolutions institutionnelles les plus récentes, qui ont élargi le champ des objectifs de l'activité de recherche à bien d'autres choses que la production de connaissances innovantes, notamment à la construction de compétences professionnelles, comme nous l'avons vu plus haut (partie 0.3). D'autre part, il nous permet ici de caractériser ce qui fait la spécificité des trois contributions que nous avons choisi de regrouper ici, celle de Christian Bonah et Joël Danet (04001) sur la formation des futurs médecins, celle de Sylvain Laubé et Grégory Chambon (04002) sur la formation d'enseignants et de médiateurs culturels, enfin celle de Katalin Gosztonyi (04003) sur la formation des futurs enseignants

\footnotetext{
52 EMEE http: //www.museums-exhibiting-europe.de/ (consulté 9.10.14).

53 En particulier d'un point de vue historique, puis J.M. Barbier oppose le premier paradigme comme le paradigme humboldtien, et le second comme franchement contemporain. Pour une lecture un peu différente de cette histoire, et de son incidence sur les liens entre recherche et enseignement, voir la contribution de Bonah et Danet (04001), notamment la note 3.
} 
de mathématiques. Elles ont en effet comme point commun de décrire dans les termes d'une expérience individuelle ou collective, en revenant généralement sur la genèse et les circonstances de projets, des démarches où les activités de recherche et de formation professionnalisantes entrent dans une dialectique constante. Ces expériences doivent ainsi se comprendre par rapport à des histoires intellectuelles et humaines impliquant des changements de point de vue fondamentaux.

\subsection{Trois parcours « d'enseignement-recherche »}

Christian Bonah et Joël Danet utilisent le terme d' « enseignement-recherche » pour insister sur l'imbrication des deux dimensions du projet MedFilm. L'enjeu originel qui a motivé ce projet de recherche est un enjeu de formation à la faculté de médecine de Strasbourg. L'enseignement développé depuis quinze ans au département d'Histoire des Sciences de la Vie et de la Santé, caractérisé par l'utilisation de vieux films « utilitaires » médicaux et sanitaires, était en effet confronté à des problèmes de contextualisation historique et pédagogique, ainsi que des problèmes de disponibilité de ces films. Ce projet sur l'histoire du film médical et sanitaire en France a été l'occasion de constituer un projet collectif appuyé sur une plateforme numérique. Cette dernière est en effet un outil de recherche pour archiver les films et structurer les travaux d'analyse des chercheurs, mais aussi outil pour la formation professionnelle des étudiants, certains d'entre eux étant impliqués in fine dans le développement du site. Cette expérience collective a donc permis de structurer un nouveau réseau de recherche en voie de développement, dont la plateforme numérique est un instrument et dont les étudiants sont partie prenante. Cette histoire n'est à son tour pas séparable des développements pédagogiques qui lui ont donné naissance, et que le projet MedFilm permet de développer en retour.

Grégory Chambon et Sylvain Laubé quant à eux décrivent les interactions entre la redéfinition progressive des activités de recherche menées au sein du Centre François Viète (antenne de l'Université de Bretagne Occidentale) et la mise au point concomitante d'une formation de master « Histoire des sciences et techniques, humanités numériques et médiations culturelles », récemment ouverte. La création de cette formation part d'un besoin en personnel spécialisé, exprimé par les partenaires du centre de recherche tels que collectivités territoriales et musées et qui rejoint le souci de professionnaliser les étudiants concernés, qu'il s'agisse de futurs enseignants ou de futurs médiateurs de la culture scientifique. Les auteurs décrivent les éléments de contenu de cette formation, au regard de compétences de la figure de «chercheur polyvalent » ainsi visée. Ils montrent ainsi comment les étudiants de ce master doivent s'engager dans une activité de chercheur directement liée à des opérations de recherche en cours au Centre François Viète, et compatible cependant avec le développement de leurs compétences professionnelles.

Enfin Katalin Gosztonyi montre comment les questions qui se posaient d'abord à elle comme jeune enseignante, pour mettre en œuvre un atelier d'été d'histoire des mathématiques auprès d'un jeune public, ont finalement motivé une recherche à cheval entre l'histoire et la didactique des mathématiques qu'elle poursuit dans le cadre d'un doctorat en cotutelle. L'une de ses motivations personnelles rejoint une conviction partagée avec ses collègues hongrois, à savoir qu'il existe une «tradition hongroise » de l'enseignement des mathématiques mais pas de caractérisation «savante » de cette tradition, ce qui rend difficile sa valorisation au plan international. La recherche qu'elle mène dans le cadre de sa thèse de doctorat contribue à cet éclaircissement par une étude historique des travaux de mathématiciens hongrois des années 1940-50 et des mouvements de réforme de l'enseignement des mathématiques des années 1960-70. Cette recherche permet non seulement de spécifier la tradition hongroise en question, mais aussi de caractériser son influence dans l'enseignement des mathématiques. L'auteure explique ensuite, dans une partie plus prospective mais déjà appuyée sur ses premières expériences en France ou en Angleterre, comment elle compte mobiliser les textes mêmes qu'elle étudie dans sa thèse comme objets d'étude pour la formation d'enseignants. On assiste là, de nouveau, au développement d'un questionnement qui articule concomitamment recherche et conception de formation. 


\subsection{Trois similitudes de ces études : leur caractère génétique, le poids des circonstances institutionnelles, le caractère interdisciplinaire des champs de recherche}

La similitude frappante du caractère génétique des trois contributions renvoie à une question plus générale que nous nous contentons de soulever ici : revenir sur une histoire et en problématiser les enjeux n'est-il pas le moyen le plus naturel de montrer comment s'articulent deux visées qu'on est le plus souvent habitué à dissocier, en raison de la différence des deux types d'activité, l'initiation à la recherche et la formation professionnelle? Alors que les informaticiens qui travaillent sur un projet sont habitués à documenter l'évolution de ce dernier, chercheurs et formateurs « documentent » beaucoup plus rarement les étapes du cheminement qui les conduit à construire un projet de recherche : cela conduit à récapituler les motivations réelles, génétiques, et qui sont d'un autre ordre que les motivations scientifiques du projet de recherche lui-même. C'est probablement au niveau de ces «motivations génétiques » que l'articulation à un projet de formation professionnelle peut se faire jour.

En outre, cette perspective génétique indique dans les trois cas présentés le poids important des circonstances institutionnelles. Ce dernier est évident dans les contributions de Bonah-Danet et LaubéChambon, qui récapitulent l'élaboration de projets ambitieux qu'ils étaient en position de mener à bien. Si des parties importantes de leurs contributions prennent l'allure de bilans de projets, c'est qu'ils doivent effectivement répondre à des sollicitations complexes de la part des institutions, où les problématiques d'insertion ou de formation professionnelles, de développement de l'université et de reconfiguration de la recherche tiennent une place centrale. Ce poids des institutions est plus discret dans la contribution de Goztonyi, mais il est bien présent au travers de la participation à des projets collaboratifs à l'échelle internationale de sorte que la conception d'une formation de caractère apparemment local est déjà à rapporter au contexte international auquel elle devra s'articuler pour gagner en légitimité.

Le récit génétique révèle encore un autre point commun très frappant, concernant les champs de recherche investis : à chaque fois, il s'agit de champs de recherches multiples, de sorte que les projets sont nettement pluridisciplinaires. Si tous ont trait en l'occurrence à l'histoire des sciences et des techniques, tous également croisent cette préoccupation avec d'autres champs pour lesquels certaines aspects liés à l'enseignement, la médiation culturelle ou la formation professionnelle constituent des objets : les humanités numériques et le champ des « visual studies » pour Bonah-Danet, la recherche en informatique sur les Environnements Informatiques pour l'Apprentissage Humain (EIAH) pour Laubé-Chambon, la didactique des mathématiques pour Goztonyi. L'articulation entre recherche et formation professionnelle est donc reflétée pour ainsi dire par le caractère interdisciplinaire et composite de la recherche elle-même. Dans le cas de Bonah-Danet et Laubé-Chambon, qui font largement appel aux outils numériques, cette articulation renvoie typiquement aux problématiques des humanités numériques, où l'on cherche - ne serait-ce que pour des questions de pérennité de projets généralement coûteux et lourds en termes de collaborations - une articulation équilibrée entre les aspects recherche, valorisation et formation.

\subsection{La question originale de l'ambivalence des objets de recherche, vus comme « objets pédagogiques transitionnels »}

Le dernier point commun qu'il faut relever entre ces trois contributions, probablement le plus profond et tout à la fois le plus subtil, tient au caractère ambivalent de l'objet de recherche et de formation à chaque fois retenu. Ce sont probablement Bonah et Danet qui vont ici le plus loin dans la théorisation de cette ambivalence, en empruntant à la psychologie dynamique et particulièrement à Donald Winnicott (1975) la notion d'objet transitionnel : ils caractérisent ainsi comme objets pédagogiques transitionnels ces objets ambivalents que sont, pour eux, les films documentaires. Ce sont à la fois les objets possibles 
d'une identification, même momentanée, entre une situation fictive et une situation professionnelle vécue, et l'objet d'une mise à distance, à laquelle concourt en l'occurrence la recherche historique. L'articulation profonde de la perspective de recherche - en l'occurrence historique et critique - et de la perspective de formation professionnelle, se noue probablement ici, dans cet objet transitionnel à au moins deux titres : pour l'étudiant dans le cadre de sa formation, pour le chercheur-enseignant qui doit concevoir ensemble recherche et formation. De même, Laubé et Chambon insistent sur l'importance du recentrage de leur programme de recherche brestois autour de la " culture matérielle », entre autres perspectives de recherche. Même si la chose n'est pas énoncée en tant que telle dans leur contribution, on peut spéculer que le choix de ce type d'approche par les artefacts n'est pas déconnecté des perspectives de formation professionnelles qu'elle offre pour les futurs enseignants et médiateurs, qui doivent s'interroger quant à eux sur la manière dont les élèves ou le grand public « investissent » certains artefacts pour en faire des objets d'appropriation ${ }^{54}$. Enfin Gosztonyi insiste sur la transition fondamentale que représente pour elle le changement de point de vue sur un même objet, passé de l'état d'inspiration pour la conception d'un enseignement à l'état d'objet de recherche. En prolongeant cette remarque, on peut à nouveau spéculer que ce basculement, d'abord éprouvé dans son parcours intellectuel par l'auteure, en fait précisément un objet « intéressant » pour la formation qu'elle imagine. Autrement dit, pour les futurs enseignants c'est précisément cette ambivalence entre « objet d'histoire » et « objet de tradition », donc aussi d'identification relative, qui en fait un objet porteur.

Ces contributions offrent donc des perspectives intéressantes, notamment parce qu'elles permettent de décaler et d'élargir le champ des objets qui semblent légitimes pour une recherche sur des questions d'enseignement et de formation. On peut risquer l'hypothèse que ces objets ne sont pas nécessairement, ou seulement, les pratiques professionnelles directement observées, mais aussi des objets décalés, comme certains artefacts historiques bien choisis, qui offrent aux futurs professionnels des objets de « projection d'eux-mêmes » en situation, et de prise de distance critique.

Ces réflexions peuvent être prolongées par la lecture, dans le même volume, de la contribution de Husson, Brechenmacher et Bernard (05004), qui proposent eux aussi une discussion de la valeur de certaines activités de recherche pour la formation continue des enseignants à caractère interdisciplinaire, en s'appuyant sur les théories de l'activité qui sous-tendent le paradigme « intégrateur » dont parle J.M. Barbier. La contribution de Charles Lenay et de ses collaborateurs (05001) ou de Guillaume Carnino (05002) permet elle aussi d'envisager des activités « intégrées » où recherche en sciences humaines et conception d'enseignement technologiques vont de pair.

\section{Fondements, enjeux et perspectives}

\subsection{La raison d'être de réflexions pluralistes sur des enjeux fondamentaux des objets étudiés dans ce volume}

C'est précisément le caractère instable et transitionnel des objets d'étude qui constituent ce volume, leur ambivalence, leur statut de questions à discuter, qui conduit tous les contributeurs à s'interroger sur la nature de leurs activités, sur les valeurs, sur les fondements et sur le cadre épistémologique qui les sous-tendent. Nous avons réuni dans cette cinquième partie quatre contributions qui n'abordent pas seulement ces questions à titre de préliminaire ou de conclusion, mais qui en ont fait le cœur de leur contribution et pointent au moins quatre occasions de questionnements autour des sciences

\footnotetext{
54 Voir à ce sujet l'argumentaire de la journée d'étude L'inscription spatiale des sciences et ses enjeux : expositions, dispositifs, architectures, récemment organisée au Musée Curie par Andrée Bergeron, Charlotte Bigg (Centre Koyré), Jochen Hennig (Humbolt Universität) et Renaud Huynh (Musée Curie) dans le cadre du séminaire Voir/Savoir du Centre A. Koyré. Pour un compte rendu, consulter http://ciera. hypotheses.org/734 (consulté 9.10.14).
} 
humaines dans les formations professionnalisantes, liste qui n’épuise bien évidemment pas tous les questionnements fondamentaux que posent ces enseignements.

a) Un premier lieu de questionnement est l'articulation entre sciences et techniques, ellesmêmes articulées aux sciences humaines et aux humanités, que proposent de repenser de manière originale Charles Lenay, Pascal Salembier, Pierre Lamard, Yves-Claude Lequin et Loïc Sauvée (05001), qui montrent à quel point la constitutivité technique croise en permanence la constitutivité sociale à la fois en sciences de l'ingénieur et en sciences humaines et sociales, notamment dans la démarche de conception.

b) Second lieu de questionnement, l'épistémologie des techniques ou d'épistémotechnique, pensée comme articulation des techniques avec le monde social. Il s'agit à la fois de montrer comment les techniques répondent à une demande sociale, mais aussi modifient profondément les usages et le monde social, comme le montre Guillaume Carnino (05002) à travers plusieurs exemples.

c) Un troisième lieu de questionnement est l'éthique pour les ingénieurs, à la fois recherche et enseignement en émergence. Guillaume Quiquerez (05003) s'interroge sur les fondements de cet enseignement dans le cadre des écoles d'ingénieurs dont il est déjà question dans les contributions de G. Aiguier et C. Didier (02003) d'une part, et de C. Colmellere d'autre part (03001) : l'auteur propose d'une manière originale de repenser les fondements à la lumière de positions philosophiques classiques (Weber, Jonas, Ricoeur) mais aussi de théories connexes d'épistémologie de l'économie (A. Sen).

d) Un quatrième lieu de questionnement est l'articulation entre les enseignements d'épistémologie et d'histoire des sciences et la formation professionnelle en tant que telle : quelles continuités ou discontinuités, quels réinvestissements possibles, ces enseignements sont-ils là simplement pour susciter une démarche plus réflexive ou apporter une «culture générale » de la discipline, ou présentent-ils de réelles potentialités de réinvestissement dans la pratique professionnelle ? Matthieu Husson, Alain Bernard et Frédéric Brechenmacher (05004) s'interrogent sur les apports et les limites des formations pluridisciplinaires en histoire des sciences offertes aux enseignants en poste : à partir des recherches sur la place de l'histoire des sciences dans l'enseignement secondaire, d'une part, et sur l'activité enseignante en butte à des dilemmes professionnels de l'autre, ils proposent une réflexion de fond sur les orientations permettant de rendre pertinentes de telles formations dans un cadre à la fois universitaire et professionnel.

Puisque ces quatre contributions entendent mettre en avant une réflexion sur les présupposés fondamentaux permettant de penser et concevoir d'une manière innovante les types de formations visées à chaque fois, il aurait été tentant d'en faire une sorte de préalable à toutes les autres. Mais cela n'aurait évidemment pas rendu justice à la richesse des questions fondamentales abordées dans les précédentes contributions, qui dépasse le cercle des questions qu'abordent ici ces quatre contributeurs. Et surtout, elle aurait donné une image réductrice et faussement «normalisante » de ces interventions, dont l'esprit reflète avant tout un remarquable et stimulant pluralisme épistémologique, associé à une volonté de définir un parti-pris argumenté apte à provoquer le lecteur plutôt que de lui donner de fausses certitudes. Ce pluralisme semble en effet plus propre à engager ou entretenir un débat autour de questions fondamentales et depuis une position particulière assumée comme telle, qu'à définir un terrain de consensus probablement inexistant, et ceci même si l'on tient compte de plusieurs points de convergence remarquables sur lesquels nous reviendront plus bas ( 55.4$)$.

La lecture comparée des contributions fait en effet apparaître bien vite que les objets de réflexion retenus de même que les points de vue, les champs théoriques ou les références retenus à chaque fois pour les aborder sont extrêmement variés. De surcroît ils partent à chaque fois d'hypothèses fortes, parfois militantes, doublé d'un état des lieux ou d'un historique lui-même présenté de manière critique, pour lequel une proposition alternative et innovante est ensuite proposée en détail. En paraphrasant et en détournant légèrement le propos de l'un des contributeurs, G. Quiquerez, on peut dire que le fait de 
conjuguer la volonté militante de provoquer le lecteur à réfléchir sur des questions fondamentales, à la volonté de n'imposer aucune valeur en particulier, «requiert de s'arrimer à une perspective éthique »et épistémologique- "authentiquement pluraliste, devant conduire à la mise au jour, à l'examen et à la discussion intersubjective des présupposés axiologiques à l'œuvre. »

\subsection{Quatre systèmes critiques débouchant sur des hypothèses fortes et de nouveaux points de vue sur la conception de formations professionnelles}

Charles Lenay et ses collègues partent d'une critique approfondie d'un modèle à leurs yeux déficient, qui est celui des approches pluridisciplinaires « classiques » qui n'articulent pas suffisamment sciences humaines et sciences de l'ingénieur. Du portrait qu'ils en font, il faut comprendre par là soit l'opposition entre deux cultures (humaniste et technique) jugées irréductibles l'une à l'autre, soit leur instrumentalisation réciproque. Ils proposent alors de redéfinir un point de convergence possible et pour ainsi dire «profond » entre ces champs, en l'espèce le «fait technique » entendu dans un sens bien précis, qui renvoie à la « constitutivitié technique de l'expérience humaine ». Ils proposent de regarder cette constitutivité technique d'une manière volontairement ambivalente à la fois comme objet possible d'étude pour des sciences humaines en contexte technologique, mais aussi, comme une manière de penser les fondements mêmes des démarches d'analyse en sciences humaines; d'où la possibilité d'un dialogue interdisciplinaire croisé entre sciences de l'ingénieur et sciences humaines, autour de cette question de leurs «fondements techniques » réciproques. Cette proposition théorique forte, dont ils font le cœur vivant d'un nouveau groupement d'intérêt scientifique (GIS) en gestation, a des conséquences pédagogiques qu'ils évoquent en conclusion : même si les exemples ne sont pas donnés ici de manière détaillée ${ }^{55}$, elle vise à revisiter le cœur de la formation des ingénieurs, à savoir le problème de la conception de procédures ou de systèmes innovants dans un contexte toujours inédit : cette démarche de conception est en effet regardée alors comme une " expérience humaine » particulière mais fondamentale et replacée au cœur d'une réflexion théorique fondée sur la recherche interdisciplinaire dont ils ont dessiné les contours.

Guillaume Carnino quant à lui part d'un historique approfondi mais là encore volontairement partisan des approches traditionnelles $\mathrm{du}$ « fait technique » dans les champs de l'anthropologie, de la sociologie et de la philosophie. Cette notion de «fait technique » est cette fois-ci entendue comme facteur possible de déterminations sociales sur le long terme. Cette compréhension part d'une critique initiale des points de vue (selon l'auteur) traditionnels en mettant en évidence la question de savoir si la technique est neutre et seuls ses usages susceptibles d'une étude critique, ou si elle est productrice d'usage sociaux sur le long terme, d'une manière qu'il s'agit alors d'étudier. C'est à ce dernier point de vue auquel l'auteur se rallie en répertoriant au passage, dans les études contemporaines, celles qui lui paraissent rejoindre cette perspective d'étude. L'ensemble de cette réflexion à la fois critique et prospective est alors replacée dans le contexte de la formation innovante développée depuis 2012 à l'université technologique de Compiègne, sous le titre « humanités et technologie ». L'enjeu est pour lui de répondre aux besoins explicitement exprimés par les étudiants de comprendre les effets à long terme de la technique pour constituer ce qu'il appelle « une grammaire de l'acte et du changement technique et humain à la fois ». L'enjeu concret est d'établir « aussi bien des terrains que des grilles d'analyse permettant de qualifier l'émergence et l'impact propre de phénomènes techniques et technologiques, sur le temps long de l'histoire et dans l'épaisseur du social ». Pour illustrer cette ambition novatrice, il développe in fine un exemple d'objet d'étude et de recherche possible pour ce cadre d'enseignement

55 Mais on peut consulter (Forest et alii, 2005). 
par la recherche, à savoir l'étude de la place et de l'impact social de l'existence des «flux de travail » ou « workflows » qui ont pris une place centrale dans les méthodes de travail et de gestion de bien des entreprises contemporaines.

Guillaume Quiquerez part quant à lui d'une discussion là encore volontairement polémique des conceptions possibles d'un enseignement d'éthique en école d'ingénieurs. En partant d'un état des lieux sur la demande sociale adressée aujourd'hui aux formations en écoles d'ingénieurs vues comme lieu privilégié de la formation des élites (françaises), il définit l'objet problématique de son questionnement en remarquant que la demande faite aux écoles d'ingénieur de dispenser une formation aux questions éthiques les place de fait dans la position d'un acteur du jeu social sommé d'engagé sa « responsabilité sociale », à l'exemple de ce qu'on exige aujourd'hui des entreprises. Après une discussion des questions classiques discutées en sociologie, l'argumentation débouche sur le compromis à établir entre l'explicitation inévitable de valeurs axiologiques dans un cadre académique, et leur relativisation dans le cadre d'un débat critique où les présupposés de ces partis pris sont explicités et discutés. Quiquerez s'engage alors lui-même davantage dans un parti-pris explicité, en opposant une stratégie dite « idéalnormative » visant à définir le portrait « idéal » d'un comportement éthique de référence, à une stratégie dite « axiologique-située ${ }^{56}$ qui vise à rapporter les débats éthiques à des situations concrètes et localisées. Ces idées sont finalement renforcées par une discussion sur les questions classiques d'éthique de la responsabilité (Weber, Jonas), mais qui prennent une couleur particulière dans le cas des activités d'ingénieur dont les conséquences proches et lointaines ne sont pas toujours faciles à délimiter et donc, à imputer en termes de responsabilité. Pour aborder ces questions délicates, l'auteur reprend à son compte les idées de Ricoeur et de Sen à nouveau, pour proposer un concept de responsabilité à la fois située et fondée sur l'idée de «prise en charge de la vulnérabilité ». G. Quiquerez dessine en creux les conséquences pédagogiques à tirer de cette réflexion d'ensemble, et on devine facilement vers quel type général de dispositif cette « mise en situation concrète » de questions éthiques peut tendre. Il souligne simplement, in fine, que ces démarches sont pour lui irréductibles à la simple acquisition de compétences professionnelles.

Enfin Alain Bernard, Matthieu Husson et Frédéric Brechenmacher posent une hypothèse forte sur les présupposés de formation continues de type universitaire en histoire des sciences qui puissent être considérées comme "viables », même si les principes d'orientation qu'ils défendent sont présentés comme consensuels. Ces hypothèses se fondent sur une double analyse. La première est historique et vise à cerner la situation paradoxale des enseignants du secondaire, partagés entre une position de spécialistes d'une discipline particulière dans un système institutionnel compartimenté, et enseignants généralistes, la question de l'enseignement de l'histoire des sciences étant précisément située à l'interface de ces deux exigences. La seconde part des recherches sur les théories de l'activité et sur le positionnement de professionnels - notamment enseignants - placés dans des situations complexes engendrant des dilemmes, qui appellent des choix et des compromis. Une des hypothèses fondamentales qui est alors faite est que les formations continues pour enseignants devraient être adaptées à cette situation très particulière des enseignants et aux complexités propres à leur action. De cette analyse ils déduisent des orientations générales pour faire des formations en question un espace de délibération où les enseignants concernés puissent élaborer collectivement les dilemmes qu'ils doivent affronter et développer leur pouvoir d'agir, selon l'expression des psychologues du travail. Plusieurs exemples sont donnés in fine de projets pédagogiques en cours, où ces idées directrices orientent les dispositifs mis en œuvre. Les auteurs soulignent en conclusion que ces projets ne touchent pas seulement à la conception des formations visées, mais aussi à celle des ressources associées à l'enseignement et de leur accessibilité.

\footnotetext{
56 Directement inspirée dans idées d'Amartya Sen sur la théorie de la justice dans un monde pluraliste.
} 


\subsection{Points de convergence et perspectives}

On a vu plus haut que la variété évidente des positionnements retenus fait par elle-même tout l'intérêt de ces contributions car elle provoque la réflexion et invite finalement à s'interroger plus généralement sur toutes sortes de questions «fondamentales » du même type. Au-delà cependant de cette variété bénéfique, on peut néanmoins relever plusieurs points communs à toutes, ou à plusieurs d'entre elles.

Tout d'abord, toutes partent d'un constat problématique sur la situation dont elles font l'état des lieux. Dans cette argumentation à chaque fois orientée, les apports de l'histoire ou de la sociologie ne sont pas convoqués pour eux-mêmes mais pour les besoins d'une démonstration précise. Ainsi Lenay et ses collègues proposent un historique qui dresse un portrait sévère des essais d'intégration d'une perspective de sciences humaines dans la formation des ingénieurs, histoire dans laquelle la fondation des universités technologiques tient évidemment le beau rôle, malgré l'échec relatif d'une tentative dont les ambitions initiales (au début des années 70) étaient nettement supérieures à ce qui existe aujourd'hui. On mettra bien sûr en regard cette histoire avec le panorama proposé par Michel Cotte dans le même volume, et en général avec la littérature existante sur l'histoire complexe de l'enseignement technologique et professionnel en France. ${ }^{57}$ De même, Carnino renvoie à un état des lieux de la sociologie et de la philosophie des techniques qui privilégie in fine la perspective qu'il a choisi lui-même d'embrasser. Il n'est reste pas moins que le champ reste le lieu de débats multiples et contradictoires, comme on pourra en juger en lisant les propos liminaires de la contribution de Colmellere et les critique que l'auteure adresse dans son introduction aux positions défendues par Bruno Latour en sociologie des sciences. Quiquerez assume quant à lui explicitement de prendre parti dans un débat d'autant plus riche qu'il est largement en gestation pour les raisons déjà relevées dans notre commentaire à la contribution d'Aiguier et Didier : on comparera donc utilement leurs perspectives, présupposés et conclusions. ${ }^{58}$ Enfin Husson, Bernard et Brechenmacher se fondent sur un historique des orientations « généralistes » de l'enseignement secondaire qui demanderait certainement d'être approfondi pour tenir compte du phénomène contemporain qu'ils relèvent au passage: la complexification des tâches des enseignants sous l'effet de la complexification du paysage institutionnel lui-même.

De manière générale, et quelle que soit la lecture historique et sociologique qu' on veut bien accorder ou contester aux contributeurs, le point commun de ces mises en perspective est de donner les moyens de penser une situation contemporaine placée sous le signe de la complexité des tâches des professionnels et de la présence toujours plus appuyée de demandes sociétales qui travaillent désormais les institutions de l'intérieur. De ce point de vue, ces réflexions peuvent être vues comme une série de réponses profondes à un paysage changeant et évolutif, rejoignant ainsi certaines des conclusions de la contribution d'AnneSophie Godfroy (01003).

Ensuite, les contributions sont toutes situées explicitement ou non, et révèlent donc à leur manière leur dépendance au contexte institutionnel qui rend ou non possible le développement de ces réflexions. On rejoint ici les problématiques déjà évoquées au sujet des contributions de la partie 2 (Influence du contexte institutionnel). La chose est très évidente pour les contributions de Lenay et ses collègues ainsi que celle de Carnino, qui ont en commun de s'inscrire dans le paysage des universités technologiques qui revendiquent depuis leur fondation une très forte composante de recherche et d'innovation autour de l'intégration des sciences humaines et sociales dans la formation des ingénieurs. Le type de réflexion à la fois critique et prospective qui nous est proposé ici est donc « traditionnel », à sa manière, si on le jauge à l'aune des attentes habituelles des universités technologiques, comme le laissait déjà entendre Michel Cotte (02001) dans le bilan raisonné qu'il a fait de son travail à l'UTBM. Dans le cas de la contribution

\footnotetext{
57 Voir par exemple (Pelpel et Troger 2001).

58 En particulier il est clair que l'approche des collègues lillois est bien davantage inspirée des courants de pensée anglo-saxons qui mettent la notion de construction de compétences professionnelles au cœur de la démarche de la formation à l'éthique, perspective que Quiquerez met explicitement en question.
} 
de G. Quiquerez, la réflexion s'inscrit dans le cadre d'une structure interne à l'Ecole Centrale de Marseille, où les questions de responsabilité sociale mise en avant dans la contribution tiennent une place essentielle. ${ }^{59}$ On pourra mettre cet environnement en regard avec le contexte de l'Ecole Centrale de Lyon évoqué dans la contribution de N. Hourcade (02002), où les questions d'enseignement de l'éthique tiennent également une place non négligeable, et de celui de l'Ecole Centrale de Paris dont traite la contribution de C. Colmellere (03001). Enfin, dans le cas de la contribution de Husson, Bernard et Brechenmacher, les perspectives ouvertes par cette réflexion, même si la chose reste implicite dans l'article, ne sont pas dissociables de l'évolution actuelle des ESPE, qui implique de renouveler la réflexion sur le rapport entre formation initiale et continue des enseignants, d'une part, et celle du rapport entre formation et recherche de l'autre (cf. §0.3). Un des points qui sont particulièrement d'actualité à cet égard, est la question de savoir comment faire entrer les enseignants dans les structures institutionnelles complexes dans lesquelles on leur demande désormais d'évoluer et de construire leur action.

Un troisième facteur de convergence rejoint les conclusions de la partie précédente : en effet au moins trois des contributions insistent sur l'importance, sinon la centralité de la démarche d'initiation à la recherche dans la démarche pédagogique qui se trouve à chaque fois recommandée. La différence est que la question est abordée ici d'un point de vue épistémologique et axiologique, plutôt que par un récit génétique indiquant les étapes d'une coconstruction, comme dans la partie précédente. La chose est évidente dans l'article de Lenay et ses collègues, qui insistent sur l'importance d'inscrire la démarche pédagogique au cœur d'une démarche de recherche interdisciplinaire ayant pour objet commun le « fait technique », et prenant comme support un travail raisonné de conception. Elle l'est encore dans le cas de Carnino, qui conclut sur la description d'un objet possible d'enseignement, qui n'est rien d'autre qu'un programme de recherche déjà assez détaillé. Enfin l'article de Husson, Bernard et Brechenmacher indique la compatibilité naturelle entre la démarche préconisée, qui vise à construire un espace d'élaboration de dilemmes professionnels, et celle d'une recherche-action qui est susceptible à la fois de favoriser ce type de questionnement et de l'inscrire dans une perspective plus large qui lui donne sens. ${ }^{60}$

Enfin, toutes les contributions de cette partie sont à leur manière militantes. Il revient évidemment au lecteur de juger jusqu'à quel point il sera convaincu ou non par chaque argumentation. Le plus important, comme on l'a vu plus haut, est que chacun en tire une utile matière à réflexion, doublée à chaque fois de références riches et abondantes. Il en ressort également qu'il est possible voire probablement nécessaire, comme l'argumentaire initial du colloque invitait les participants à le faire (cf. §0.2), d'adopter un point de vue critique sur les enjeux et les modalités de pratiques de formation qui, sans cela, paraîtraient banales ou allant de soi. Ce que ces contributions largement prospectives indiquent, c'est que la réflexion sur la place, la nature et le rôle des sciences humaines dans le contexte de formation professionnelle dans le domaine scientifique et technologique est bien vivante et doit le rester.

\section{Références}

[1] Albe, V. 2009. Enseigner des controverses. Rennes : PUR.

[2] Astofli, J-P. 1994 (1ère éd. 1992). L'école pour apprendre. Paris : ESF.

[3] Barbier, Jean-Marie, and Françoise Clerc. 2008. «Formation et recherche ? : ambiguïtés sémantiques et formes d'action spécifiques. » Recherche et formation, no. 59 (Décembre) : 133-40. doi:10.4000/rechercheformation.662.

\footnotetext{
59 Voir la page dédiée sur le site de l'école centrale de Marseille: https://www.centrale-marseille.fr/fr/page/ responsabilite-societale (consulté le 28.10.2014).

${ }^{60}$ La contribution de Quiquerez est probablement celle qui est la moins explicite sur le programme de recherche sous-jacent aux réflexions proposées, et sur la manière dont les étudiants y seraient ou seront associés ; néanmoins la conclusion laisse clairement entrevoir que la démarche pédagogique sera avant tout une démarche de questionnement qui semble bien conciliable avec une formation par la recherche.
} 
Les sciences humaines dans les parcours scientifiques et techniques professionnalisants

[4] Chang, H. 2011. "How Historical Experiments Can Improve Scientific Knowledge and Science Education: The Cases of Boiling Water and Electrochemistry." Science and education 20 (3-4): 317-341.

[5] Colet, N. R., McAlpine, L., Fanghanel, J., \& Weston, C. (2011). Le concept de Scholarship of Teaching and Learning. Recherche et formation, (67), 91-104. doi:10.4000/ rechercheformation. 1412

[6] Byk, C. 2014. La formation des scientifiques à l'éthique. Bordeaux: LEH éditions. http: //www . leh.fr/edition/science-ethique-et-societe/la-formation-des-scientifiquesa-l-ethique-9782848745381.html

[7] Derouet, A. 2013. «De l'honnête homme au manager ? La contribution des enseignements juridiques de l'École centrale à la définition d'un ingénieur d'élite depuis 1829 » Droit et société 83/1, pp. 33-47.

[8] Dietmar, Hottecke. 2000. "How and What Can We Learn From Replicating Historical Experiments? A Case Study". Science and education 9 (4): 343-362.

[9] Forest, J., Méhier, C., Micaëlli, J.P. (dir) 2005. Pour une science de la conception. UTBM.

[10] Grelon André, 2007, "French Engineers: Between Unity and Heterogeneity". History of Technology 27, 107-124.

[11] Heering, P. 2007. "Public experiments and their analysis with the replication method". Science and education 16 (6): 637.

[12] Heering, P., Wittje, R. 2011. Learning by Doing. Stuttgart: Franz Steiner Verlag.

[13] Lahire, B. 2004. À quoi sert la sociologie? Paris: La Découverte.

[14] Dubet, F. 2011. A quoi sert vraiment un sociologue? Paris: A. Colin.

[15] Le Goff, J.P. 1996. Les illusions du management : pour le retour du bon sens. Paris: La découverte.

[16] Legardez, A., Simonneaux, S. 2006. L'école à l'épreuve de l'actualité. Enseigner des questions vives. Paris: ESF

[17] Lhoste, Y. 2008. Problématisation, activités langagières et apprentissage dans les sciences de la vie. Étude de quelques débats scientifiques dans la classe dans deux thèmes biologiques : nutrition et évolution. Thèse sous la direction de C. Orange \& M. Jaubert. Université de Nantes? http://archive.bu . univ-nantes.fr/pollux/show . action?id=258bf74a-d465-4daf 941f-81d5d5576af4.

[18] Orange, C. 2012. Enseigner les sciences : Problèmes, débats et savoirs scientifiques en classe. Paris : De Boeck

[19] Pelpel, P., Troger, V. 2001. Histoire de l'enseignement technique. Paris: L'Harmattan.

[20] Rocard, M. 2007. L'enseignement scientifique aujourd'hui : une pédagogie renouvelée pour l'avenir de l'Europe. Site de la Commission Européenne : http://ec.europa.eu/ research/science-society/document_library/pdf_06/report-rocard-on-scienceeducation_fr.pdf

[21] Simonneaux, L. 2003. "L'argumentation dans les débats en classe sur une technoscience controversée ». Aster 37, 189-214. DOI: 10.4267/2042/8825

[22] Sonntag, Michel. 2007. « Les formations d'ingénieurs. Des formations professionnelles et professionnalisantes. Orientations, contenus, contextes. " Recherche et formation, no. 55 (October): 11-26.

[23] Visier, L. 2011. «Vingt ans d'enseignement des sciences humaines et sociales dans les études médicales en France ». Bioethica Forum, pp. 143-148.

[24] Von Schomberg, Rene. 2013. "A vision of responsible innovation”. In: R. Owen, M. Heintz and J Bessant (eds.) Responsible Innovation. London: John Wiley.

[25] Winnicott, D. W. 1975. Jeu et réalité : l'espace potentiel. Trad. C. Monod et J.-B. Pontalis, préface de J.-B. Pontalis. 